\title{
Isotopic fluid changes in a Neoproterozoic porphyry-epithermal system: The Uruguay mine, southern Brazil
}

\author{
Christophe Renac ${ }^{\mathrm{a}, *}$, André Sampaio Mexias ${ }^{\mathrm{b}}$, Márcia Elisa Boscato Gomes ${ }^{\mathrm{b}}$, Luiz Henrique Ronchi ${ }^{\mathrm{c}}$, \\ Lauro Valentin Stoll Nardi ${ }^{b}$, Jorge Henrique Laux ${ }^{\mathrm{d}}$ \\ a Géoazur, UMR 7329, CNRS, Bât. 1, 250 rue Albert Einstein Sophia Antipolis, 06560 Valbonne, France

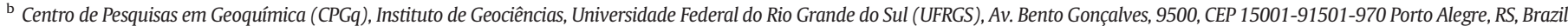 \\ c UFPEL, Praça Domingos Rodrigues n², CEP 96010-440, Pelotas, RS, Brazil \\ d CPRM, Geológical Survey of Brazil, Rua Banco da Província, 105, Bairro Santa Tereza, Porto Alegre, RS, Brazil
}

\section{A R T I C L E I N F O}

\section{Article history:}

Received 30 December 2012

Received in revised form 12 October 2013

Accepted 24 December 2013

Available online 15 January 2014

\section{Keywords:}

Copper deposits

Clay minerals

Stable isotopes

Fluids

\begin{abstract}
A B S T R A C T
Identifying the source, origin and time of emplacement of copper and gold deposits in the Neoproterozoic volcano-sedimentary and plutonic series of southern Brazil is a longstanding research goal. In this geological context, the Camaquã and Lavras do Sul areas are reported to be a post-collisional domain related to the BrazilianoPan-African Orogenic Cycle. This study focused on the Uruguay open-pit mine through a detailed assessment of the mineralogy, geochemistry and ( $, 0, \mathrm{H}$ and $\mathrm{C})$ stable isotopes of pyrite, chalcopyrite, clay minerals and carbonates as veins or disseminated sulfides in sedimentary rocks. The results indicate that the Camaquã mine is a low-sulfidation epithermal-type deposit with sulfide precipitations related to a mixture of magmatic $\left(\delta^{34} \mathrm{~S}=\right.$ 1 to $4 \%$; $\delta^{18} \mathrm{O}: 3$ to $9 \%$ ) and meteoric waters $\left(\delta^{18} \mathrm{O}=-4\right.$ to $-12 \%$ ) and a temperature varying from 350 to $80{ }^{\circ} \mathrm{C}$. Ore precipitation is associated with sequential sulfide crystallization exhausting the $\mathrm{S}$ magmatic source.
\end{abstract}

(c) 2014 Elsevier B.V. All rights reserved.

\section{Introduction}

In the Rio Grande do Sul (RS), the southernmost state of Brazil, gold mining occurs in disseminated gold deposits associated with Neoproterozoic volcano-sedimentary sequences and monzonitic to granodioritic intrusions of shoshonitic affinity related to the Lavras do Sul granitoids (Lima and Nardi, 1992, 1998; Nardi and Lima, 1985, 1988; Fig. 1). The Camaquã Basin consists of these volcanosedimentary deposits located on the eastern side of the Caçapava do Sul and Lavras do Sul granitoids (Fig. 1). On the rim of the Camaquã Basin, tens of small $\mathrm{Cu}-\mathrm{Au}-\mathrm{Pb}-\mathrm{Zn}$ deposits are disseminated. The Minas do Camaquã mine, with the Santa Maria, Uruguay and São Luiz pits, was exploited from the beginning of 1856 to 1996 and contained the largest $\mathrm{Cu}$ deposits of the basin. The copper mineralization ( $\mathrm{Cu}, \mathrm{Au}$ and $\mathrm{Ag}$ ) in the Uruguay mine has been the focus of several studies (Bettencourt, 1972; Laux et al., 2005; Leiz and Almeida, 1941; Ribeiro et al., 1966; Ronchi et al., 2000; Teixeira and Gonzáles, 1988; Veigel, 1989; Veigel and Dardena, 1990). The ore deposits are in continental sedimentary units with intercalated volcanic rocks. The main ore minerals are pyrite, chalcopyrite, bornite and hematite (Laux, 1999; Laux and Lindenmayer, 2000a,b; Ronchi et al., 2000). In previous studies, syn-depositional to diagenetic transformations of sediments produced by fluid circulation under high to low temperatures were identified. Late-stage ore was recognized through

\footnotetext{
* Corresponding author.

E-mail address: renac@unice.fr (C. Renac).
}

clay-mineral neoformation, calcite and barite, in oxidized cap-rock (Laux, 1999; Laux and Lindenmayer, 2000b; Ronchi et al., 2000).

Several genetic models have been used to explain metal enrichment of $\mathrm{Cu}, \mathrm{Pb}, \mathrm{Zn}$ and $\mathrm{Au}$, such as recurrent fracturing and compositional changes in the mineralizing fluids, either associated with "syngeneticsedimentary" and basinal fluids or magmatic fluids. Sedimentary models were proposed to explain the enrichment of the $\mathrm{Cu}$ and $\mathrm{Pb}$ ores of the Camaquã Basin sedimentary units proposed by Santos and Chaban (1978). Magmatic genetic models, based on rare earth element geochemistry (Laux, 1999; Paim and Lopes, 2000) and S and Pb isotopes (Remus et al., 1997, 1999, 2000) were proposed to explain the main ore zone of the Camaquã mine. Other studies (Laux et al., 2005; Troian et al., 2009) suggested that several hydrothermal fluids were associated with low-sulfidation mineralization (c.f. Hedenquist and Lowenstern, 1994).

The Uruguay mine consists of pyrite and chalcopyrite veins that are discordant with local sedimentary rocks (Fig. 2). The sedimentary rocks show strong hydrothermal alteration with silicification, hematite and clinochlore as alteration halos around massive ore veins and veinlets (Bettencourt, 1972; Gonzalez and Teixeira, 1980; Laux, 1999; Laux et al., 2005; Teixeira, 1978; Teixeira et al., 1978b; Veigel, 1989). Electron microscopy and crystallographic and chemical study of clay minerals (Troian et al., 2009) led to the identification of several types of chlorite (Ib90 ${ }^{\circ}$ to IIb Mg-clinochlore and Fe-chamosite polytype) and smectite (Veigel, 1992) as coatings of detrital quartz and feldspars, filling primary or secondary porosities or veins. Remus et al. $(1999,2000)$ have emphasized the magmatic origin of sulfides and described the paragenetic sequence of mineralization. 


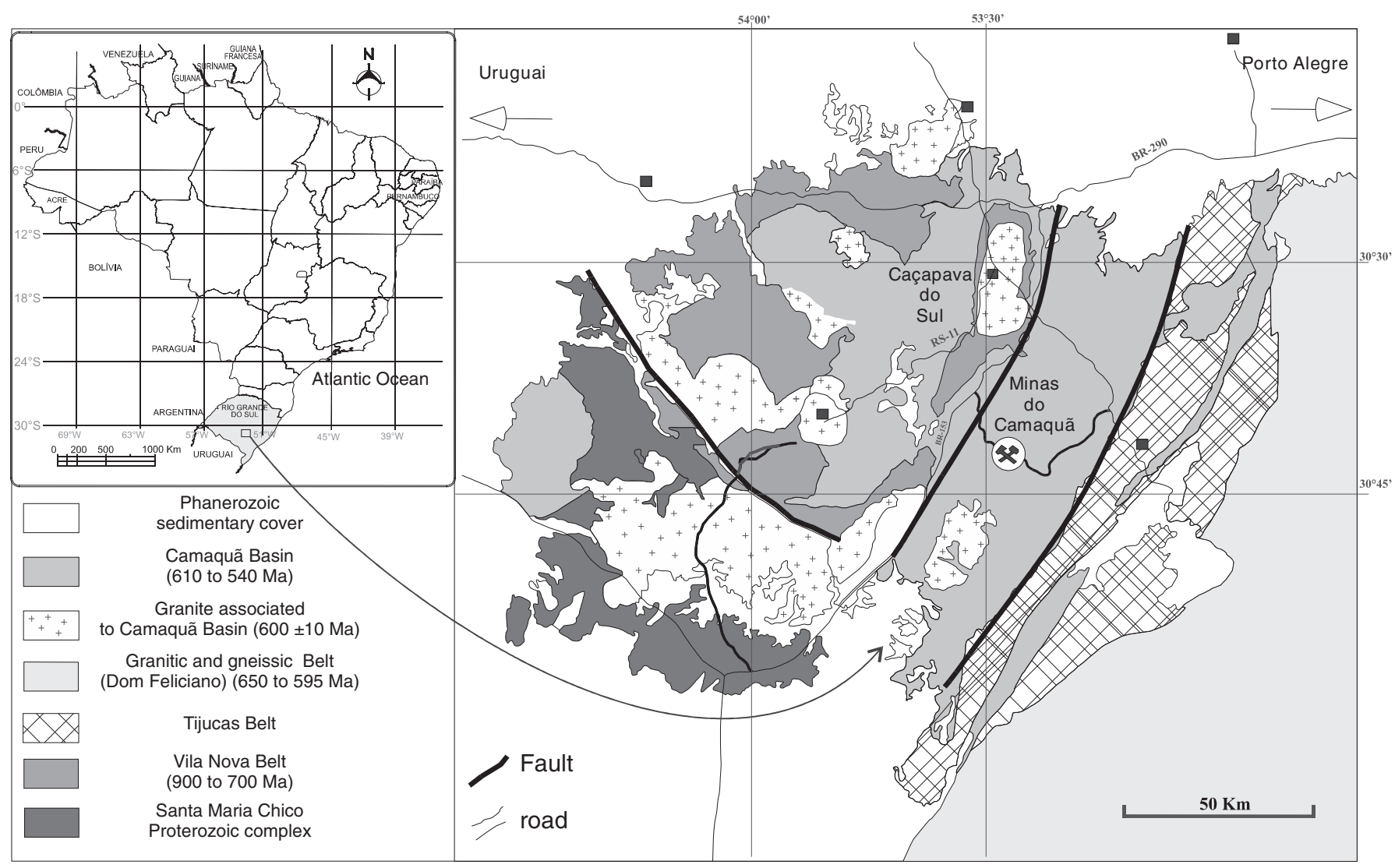

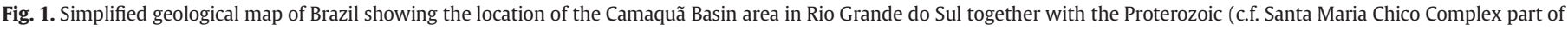
Southern Brazilian Shear Belt) and Phanerozoic sedimentary cover. Rectangle represents the location of the studied mining area.

This study discusses the crystallographic and geochemical changes related to the origin of fluids and the genetic models, roles of fluid circulation and water/rock interaction in veins and porous sandstones associated with the ore in the Uruguay mine.

\section{Regional geological setting}

The Camaquã Basin contains sedimentary and volcanic rocks of Neoproterozoic age covering approximately $10 \%$ of the Sul-RioGrandense Shield area in southernmost Brazil (Fig. 1). The volcanic and sedimentary sequences lie over older igneous-metamorphic associations composed of Paleoproterozoic metamorphic sequences with ages older than $650 \mathrm{Ma}$ (c.f. Basei et al., 2000; Bitencourt and Nardi, 2000; da Silva et al., 1999; Fragoso-Cesar, 1980; Fragoso-César et al., 1992; Hartmann, 1998; Ribeiro and Fantinel, 1978). According to Brito Neves and Cordani (1991), the Neoproterozoic geotectonic setting in southernmost Brazil involved the subduction and closure of an oceanic basin by the collision and amalgamation of at least five large continental masses. The main Braziliano-Pan-African collision in southernmost Brazil probably occurred at $650 \mathrm{Ma}$ (Gross et al., 2006; Philipp et al., 2009). Geochronological data from magmatic rocks in the Camaquã Basin indicate ages from 620 to 537 Ma (Almeida et al., 2005) with a minimum age of $470 \mathrm{Ma}$ (Hartmann et al., 2007). The Camaquã Basin magmatism and sediment deposition occurred simultaneously with the development of the Southern Brazilian Shear Belt in the eastern part of Sul-Rio-Grandense Shield (Fig. 1). This Southern Brazilian Shear Belt was an important conduit for magma transport and emplacement, and it was responsible for mantle decompression and reactivation of pre-existing magmatic sources (Bitencourt and Nardi, 2000; Nardi and Bitencourt, 2009). The Camaquã Basin is, therefore, considered by several authors to be a post-collisional strike-slip basin (Leites et al.,
1990; Oliveira and Fernandes, 1991; Wildner et al., 1999), although others (c.f. Fragoso-César et al., 1992) have interpreted it as an extensional post-orogenic and pre-cratonic rift system or as a back-arc basin (Chemale, 1997, 2000). Borba (2006) recognized the following three main stages in the evolution of the Camaquã Basin: the oldest corresponds to the Maricá Formation (ca. 630 to $600 \mathrm{Ma}$ ), which was developed under the compressive tectonics of the Braziliano-PanAfrican Orogeny; the second, related to the Bom Jardim Group (ca. 600 to $590 \mathrm{Ma}$ ), was also related to compressive tectonics, and the third is represented by the Camaquã group (ca. 590 to $537 \mathrm{Ma}$ ), which is associated with deposition in a pull-apart or rift basin with dominant extensional tectonics.

Magmatism in the Camaquã Basin evolves from rocks with shoshonitic affinity, with ages of ca. $600 \pm 10$ Ma for volcanic and plutonic rocks related to sodic alkaline silica-saturated series (Almeida et al., 2005; Lima and Nardi, 1998; Sommer et al., 2006; Wildner et al., 2002). The sedimentary rocks evolve from environments that represent shallow marine to lacustrine-alluvial settings to a typical continental environment according to Paim et al. (2000).

Sedimentary units of the Camaquã Basin are shown in Figs. 2 and 3. They consist of Maricá Group with marine turbidite to delta and fluvial deposits lying on magmatic to metamorphic basement rocks (Borba et al., 2007). The Maricá Group is overlaid by the Bom Jardim Group, which is composed of Vargas and Mangueirão members, also part of the Arroio dos Nobres Formation according to Paim et al. (2000), and the Lavras do Sul Shoshonitic Association (600 to $590 \mathrm{Ma}$ ). On the Bom Jardim Group lies the Acampamento Velho Group (Almeida et al., 2002, 2003; Janikian, 2004; Janikian et al., 2003), which contains volcanic and hypabyssal rocks of sodic alkaline affinity (Sommer et al., 2006; Wildner et al., 2002) and the Santa Fé Formation with its sedimentary rocks of alluvial origin. The younger Santa Barbara Group consists of 


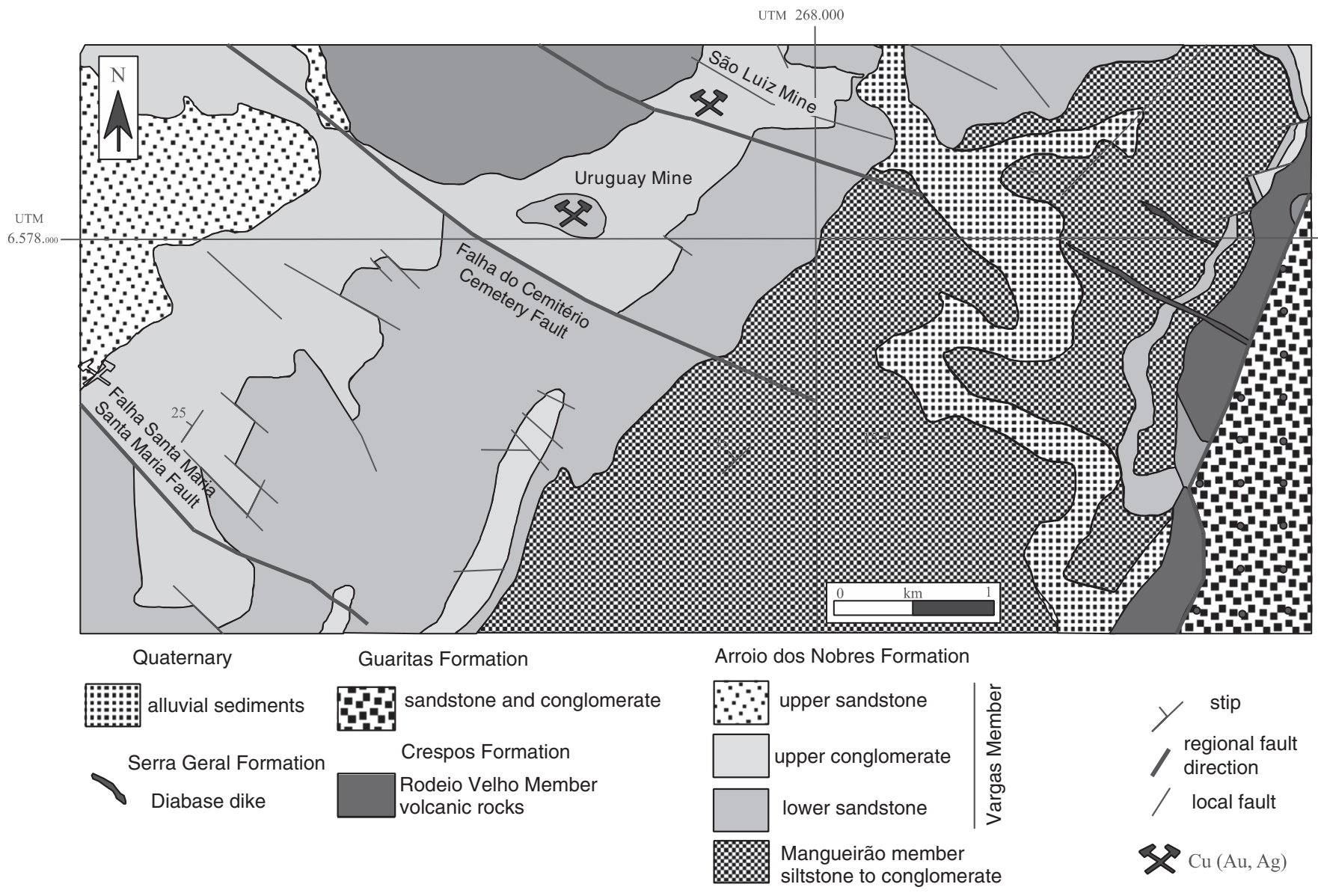

Fig. 2. Map of the southeastern part of the Camaquã Basin with the location of underground (São Luiz) and open pit mines (Uruguay).

The stratigraphy of Camaquã Basin area represented in Figs. 2 and 3 uses data from Paim et al. (1995, 2000), Ribeiro et al. (1966) and Wildner et al. (2002).

sedimentary rocks of continental deltaic origin, and the Guaritas Group. The Guaritas Group is characterized by fine-grained lenticular bodies of sandstone (Janikian, 2004; Janikian et al., 2005, 2008) with andesites and trachyandesites with ages ranging from $537 \mathrm{Ma}$ (Almeida et al., 2005) to a minimum age of $470 \mathrm{Ma}$ (Hartmann et al., 2007).

\section{The mining area and its sedimentological setting}

The Camaquã copper mine area (Bettencourt, 1972) is located at the southern part of the Camaquã Basin, in the region of Caçapava do Sul. The surface was an oxidation zone where the ore contains small amounts of gold and silver (Bettencourt, 1972). Underlying this cap rock, sedimentary units belong to the Vargas and Mangueirão members (Remus et al., 2000; Teixeira et al., 1978a,b; Figs. 3 and 4). In the Camaquã mining area, the Vargas and Mangueirão members consist of the following four levels: lower levels of sandstone, conglomerates and feldspar-rich sandstones and the upper conglomerate (Figs. 4B, 5A and $\mathrm{B}$ ). The upper conglomerate grades from rhythmically coarsepebbly sandstones to interstratified decimeter-scale gravel and sandstone. The four sedimentary levels of Camaquã's mine host boulders and gravels of reworked granitic-gneissic rocks, with a few rhyolite and pyroclastic fragments. Immature sandstones contain angulose detrital grains of quartz and microcline with less minor biotite and muscovite flakes.

\section{Ore mineralogy and its alteration halo}

The main $\mathrm{Cu}$-(Au)-rich ore zone is in meter-width veins located in northwestern dipping fractures ( $\mathrm{N} 50^{\circ} \mathrm{W}$ and $\mathrm{N} 80^{\circ} \mathrm{SW}$ ) crosscutting the sandstone and conglomerates (Ribeiro et al., 1966; Teixeira and
Gonzáles, 1988; Figs. 2 and 4C). Previous studies described intermediate and late stage magmatic ore with successive precipitation of chalcopyrite and pyrite replacing bornite and chalcopyrite, respectively (Laux, 1999; Laux and Lindenmayer, 2000a,b; Leiz and Almeida, 1941; Ronchi et al., 2000). A porphyry-type copper deposit was suggested due to the occurrence of molybdenite (Laux, 1999) and andesite in the area and the $\mathrm{Pb}$ and $\mathrm{S}$ isotopes (Remus et al., 1999) that indicate a base metal magmatic origin. This porphyry-type deposit was either related to intrusive granitoids or to the volcanic country rocks formed at the end of the Braziliano Cycle (Bettencourt, 1972; Laux et al., 2005; Remus et al., 1999, 2000; Teixeira and Gonzáles, 1988). Moreover, conglomerate sandstone and lower intermediate sandstone surrounding the main ore zone also contained disseminated sulfide grains (Figs. 3 and 4).

Laux et al. (2005), Ronchi et al. (2000) and Troian et al. (2009) have described the mineralogy in the alteration halo around $\mathrm{Cu}(\mathrm{Au})$-rich ore veins as a sequence of silicified and Fe-chamosite and Mg-clinochlore precipitations. Fluid temperatures and compositions were determined from Mg-clinochlore thermometry to be 330 to $268{ }^{\circ} \mathrm{C}$ (Lima, 1998) using thermometer developed by Cathelineau (1988). Consequently, chlorite precipitation was associated with ore deposition or late hydrothermal fluids. These temperature determinations were completed by fluid inclusion microthermometry in quartz ( 180 to $120^{\circ} \mathrm{C}$; salinity: 19.2 to $1 \mathrm{NaCl}$ weight \%) and barite $\left(<80{ }^{\circ} \mathrm{C}\right.$; salinity: 17 to $0.7 \mathrm{NaCl}$ weight \%; c.f. Lima, 1998; Ronchi et al., 2000). The large range of obtained data on temperature, salinity, chemical and isotopic values led to the proposition of several ore genetic models with different hydrothermal fluids, a Red Bed model and the epithermal/hydrothermal origin of fluids (e.g., Hedenquist and Lowenstern, 1994; Ohmoto and Goldhaber, 1997; Santos and Chaban, 1978). 


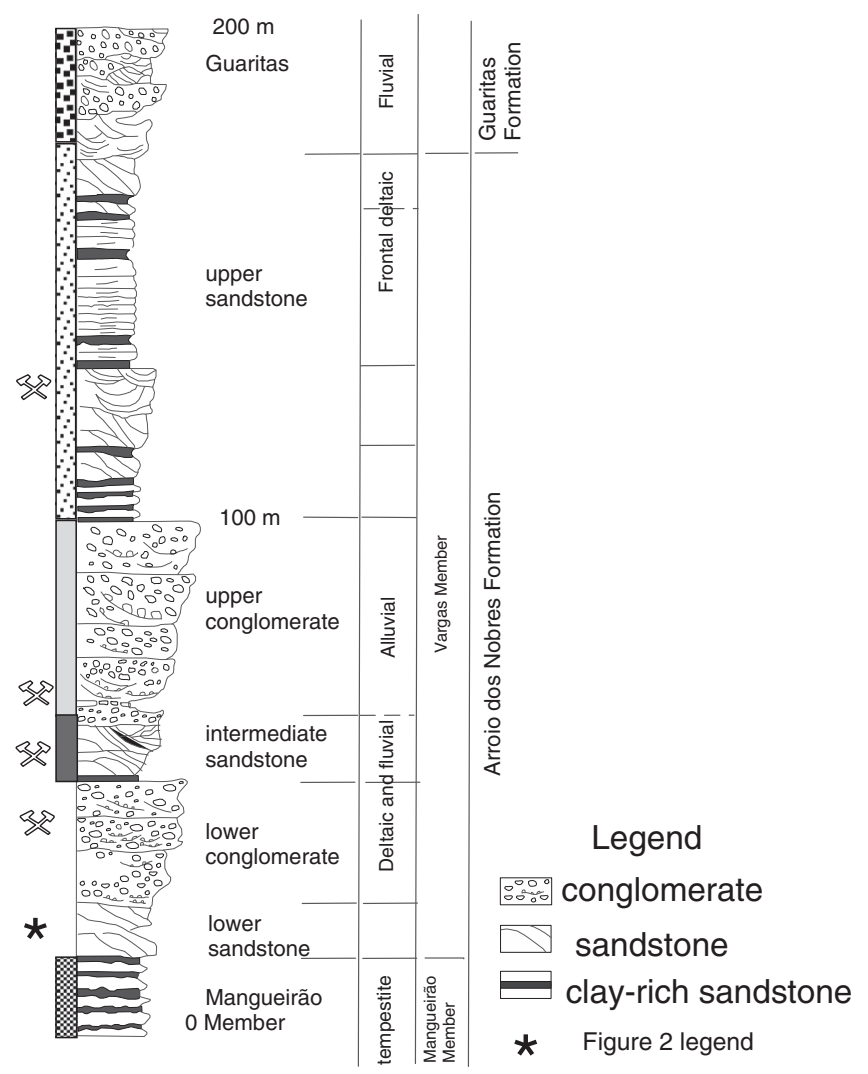

Fig. 3. A synthetic stratigraphic column is presented with sedimentological settings. Adapted from Faccini et al. (1987).

\section{Sampling strategy and methods}

The Uruguay and Sao Luiz mines were exploited as open pits. The upper part of these mines was removed, and upper sedimentary units are available through the drill core repository (Company's Brazilian Copper: $\mathrm{CBC}$ ). Five holes that were drilled prior to or during mining operations were provided by the VOTORANTIN metal company, who also provided some samples studied by Ronchi et al. (2000) (PSU-46: PTG samples). Drill cores U180/94-30 drilled in sandstones, PSU-45 in conglomerates U-80 and U-72 in intermediate sandstones were examined in the field, and thirty core samples were collected together with twenty outcrop specimens (Fig. 4). The sampling strategy was to collect and to compare the sulfide-rich samples to the less mineralized ones. Minerals were observed through macroscopic and thin section observation with optical and scanning electron microscopy. Petrographic observations by reflected light (thin and thick sections) were combined with image processing (Image J software) to measure the surface proportion $\left(>0.2 \mu \mathrm{m}^{2}\right)$ of pyrite, chalcopyrite, bornite and chalcocite prior to $\delta^{34} \mathrm{~S}$ measurements (see later methods). Microscopic observations were completed by qualitative chemical compositions of minerals (SEMEDS: Jeol JSM-5800 and Jeol JSM-5600 LV; $15 \mathrm{kV}$ and 0.6-0.7 nA with a $\mathrm{Si}-\mathrm{Li}$ diode) and quantitative analyses with an Electro microprobe (Cameca SX100-WDS, University of Jussieu; $20 \mathrm{kV}$ and 4 to $6 \mathrm{nA}$ ). Electron microprobe data of chlorite used XRD pattern information. XRD patterns of chlorite indicate a Mg and Fe octahedral occupancy with dominant $\mathrm{Fe}^{2+}$ compared to $\mathrm{Fe}^{3+}$. Consequently, the electronic mass balance is related to the $\mathrm{Fe}^{3+} / \mathrm{Fe}_{\text {tot }}$ ratio between 0.1 and 0.2 . These $\mathrm{Fe}^{3+} / \mathrm{Fe}_{\text {tot }}$ proportion induce in a total deficit lower than 0.83 for 12 octahedral charges.

For further mineralogical and geochemical analyses, grains were extracted by gentle desegregation, hand-picking, settling (clay particles: $<10,<5$ and $<2 \mu \mathrm{m}$ ) or magnetic field separation. The mineralogy of separates was checked by X-ray diffraction (XRD; Brucker Siemens
D5000 Model with $40 \mathrm{kV}$ and $25 \mathrm{~mA}$ ). Size fraction of clay particles $(<10,<5$ and $<2 \mu \mathrm{m})$ was identified by XRD with air-dried oriented, glycol-saturated and heated $\left(500{ }^{\circ} \mathrm{C}\right.$ for $2 \mathrm{~h}$ ) and powdered preparations. XRD allowed crystallinity measurements, polytype identification, estimation of dioctahedral or trioctahedral occupancy ( $(00$ l) peaks and (060) band), and Fe octahedron occupancy of clay separates (c.f. Troian et al., 2009).

Stable isotopes were determined in Saint Etienne using standard techniques for oxygen, and hydrogen stable isotopes were extracted from silicates ( $\mathrm{O}, \mathrm{H}$ : chlorite; O: quartz, hematite; Bigeleisen et al., 1952; Clayton and Mayeda, 1963; Renac et al., 2010). Carbon and oxygen were obtained after extraction, in vacuum, from $\mathrm{CO}_{2}$ carbonate by reaction with phosphoric acid at $25{ }^{\circ} \mathrm{C}$ (Craig, 1953). The extracted and purified gasses $\left(\mathrm{H}_{2}, \mathrm{CO}_{2}\right)$ were then analyzed on a gas-source mass spectrometer (Isoprime). The isotopic results are expressed as $\delta^{13} \mathrm{C}, \delta^{18} \mathrm{O}$ and $\delta^{2} \mathrm{H}(\%)$ relative to the international standard V-PDB (Vienna-Pee Dee Bellemnite) for $\delta^{13} \mathrm{C}( \pm 0.2 \%, 2 \sigma)$, and V-SMOW (Vienna-Standard Mean Ocean Water; Coplen, 1988) for $\delta^{18} \mathrm{O}( \pm 0.05$ for carbonates and $\pm 0.3 \%$ for silicates, $2 \sigma)$ and $\delta^{2} \mathrm{H}( \pm 3 \%$, $2 \sigma)$. $\delta^{34} \mathrm{~S}$ values (V-CDT: Vienna-Canyon Diablo Troilite; $\pm 0.3 \%$, $2 \sigma$ ) were measured using a Vario Micro + ISOPRIME (EA-C-IRMS: Elemental Analyzer-continuous flow-Inlet Ratio Mass-Spectrometer) with an infrared detector that allowed the measurement of S contents. Sample weights varied from 50 to $350 \mu \mathrm{g}$ and consisted of fragments from cmsize pyrite, chalcopyrite, bornite and barite. Small grains of sulfide ( $\varnothing 30 \mu \mathrm{m}, 50$ to $150 \mu \mathrm{g}$ ) in sandstone were analyzed for chemical composition (electron microprobe) and image processing (proportion of different sulfides) was also performed for thick sections for $\delta^{34} \mathrm{~S}$. The oxygen isotopes of the barite sulfate were reacted with $\mathrm{BrF}_{5}$ (Clayton and Mayeda, 1963), and the resulting $\mathrm{CO}_{2}$ gas was analyzed. For the $\mathrm{BrF}_{5}$ method, corrections were made for incomplete oxygen yield following Wasserman et al. (1992). A suite of barium sulfate standards was analyzed by all three oxygen isotope methods to ensure that equivalent results were obtained for unknowns.

\section{Results}

\subsection{Petrography and mineralogy}

Non-contiguous grains in the sandstones are surrounded by a clay matrix composed of chlorite (green and $<50 \mu \mathrm{m}$; Fig. 5C), muscovite flake and illite (Fig. 5D; Table 1) and a small amount of euhedral epidote $(<25 \mu \mathrm{m})$, some of which is associated with quartz overgrowths or calcite and Mg-rich calcite cement (Table 2). Conglomerates are composed of decimeter-sized pebbles of granite and gneisses (Fig. 5A; Table 1). Sandstones and conglomerates are cemented by chlorite (dark green), a few large crystals of chloritized biotite, illite-muscovite distorted or occasional euhedral epidote crystals $(<25 \mu \mathrm{m})$, some of which are associated with quartz overgrowths. Opaque grains are hematite and sulfides, located at grain boundaries in conglomerates. Hematite grains $(\emptyset<100 \mu \mathrm{m})$ are deposited parallel to sedimentation, whereas euhedral sulfide grains $(\emptyset<2 \mathrm{~mm})$ are disseminated in the cement or mm wide veins (Fig. 5E). These sulfide grains consisted of pyrite, chalcopyrite (Fig. 5F) and/or bornite (Table 2). Veins are filled by barite with small proportions of euhedral quartz (Fig. 5G, H). Few barite veins contain specular hematite $(<100 \mu \mathrm{m})$ or pyrite, and chalcopyrite (mm size). In the residual ore zone, located in the lower part of the Uruguay open pit (CAM 11; Fig. 4), there are decimeter wide veins filled by pyrite and chalcopyrite. This residual ore zone is surrounded by breccia with chlorite, hematite and quartz.

Petrographic observation indicates that the hydrothermal phases in sandstone and conglomerate that host the Uruguay's ore are dominantly composed of chlorite, muscovite-illite and quartz. Chlorite appears either as oriented flake coating grain fringes, filling intergranular grain or replacing biotite flakes (e.g. Walker et al., 1978). Chlorite habit is "rosette" shape with sub-hexagonal flake shape ( $<5$ to $20 \mu \mathrm{m}$ length 

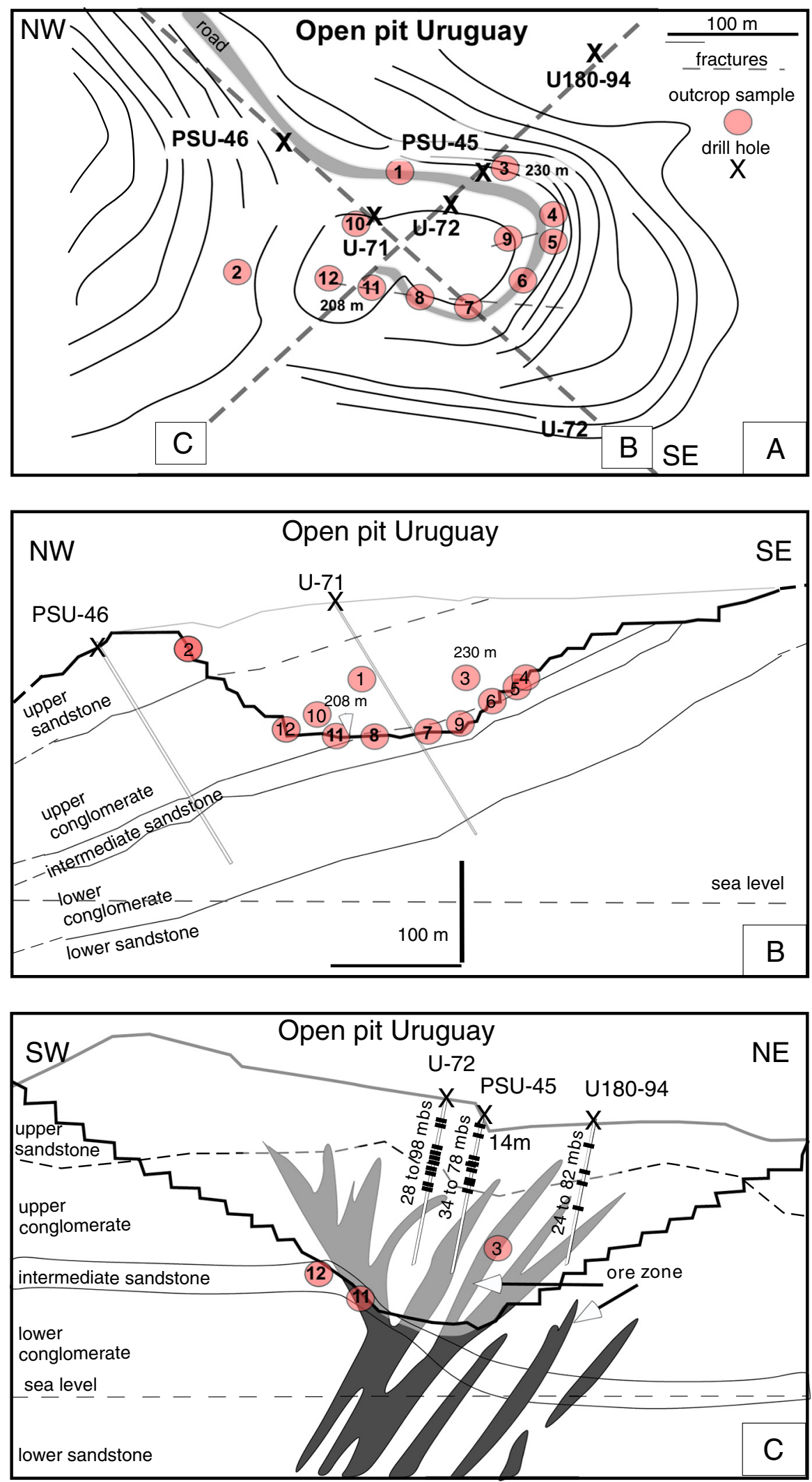

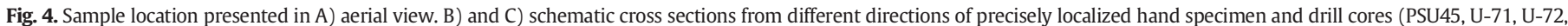
U-80, U-180-94-30; the two shades of gray indicate the former position of the main ore zones).

flake; Fig. 5C). Nearby the ore, chlorite is located in vein networks, in some cases with euhedral quartz ( $\mathrm{mm}$ size) with overgrowth underlined by primary fluid inclusion or small hematite inclusions (Fig. 5G). Similar to previous studies (Laux et al., 2005; Ronchi et al.,
2000; Troian et al., 2009) clay-size separates were Fe-chamosite and Mg-clinochlore precipitations (Bailey, 1980; Bayliss, 1975; Foster, 1962; Hayes, 1970; Table 1) with $\mathrm{Ib}^{\circ} 0^{\circ}$ to IIb polytypes (Bailey and Brown, 1962) (pure Mg-Fe-chamosite (IIb polytype; PSU45-14), 

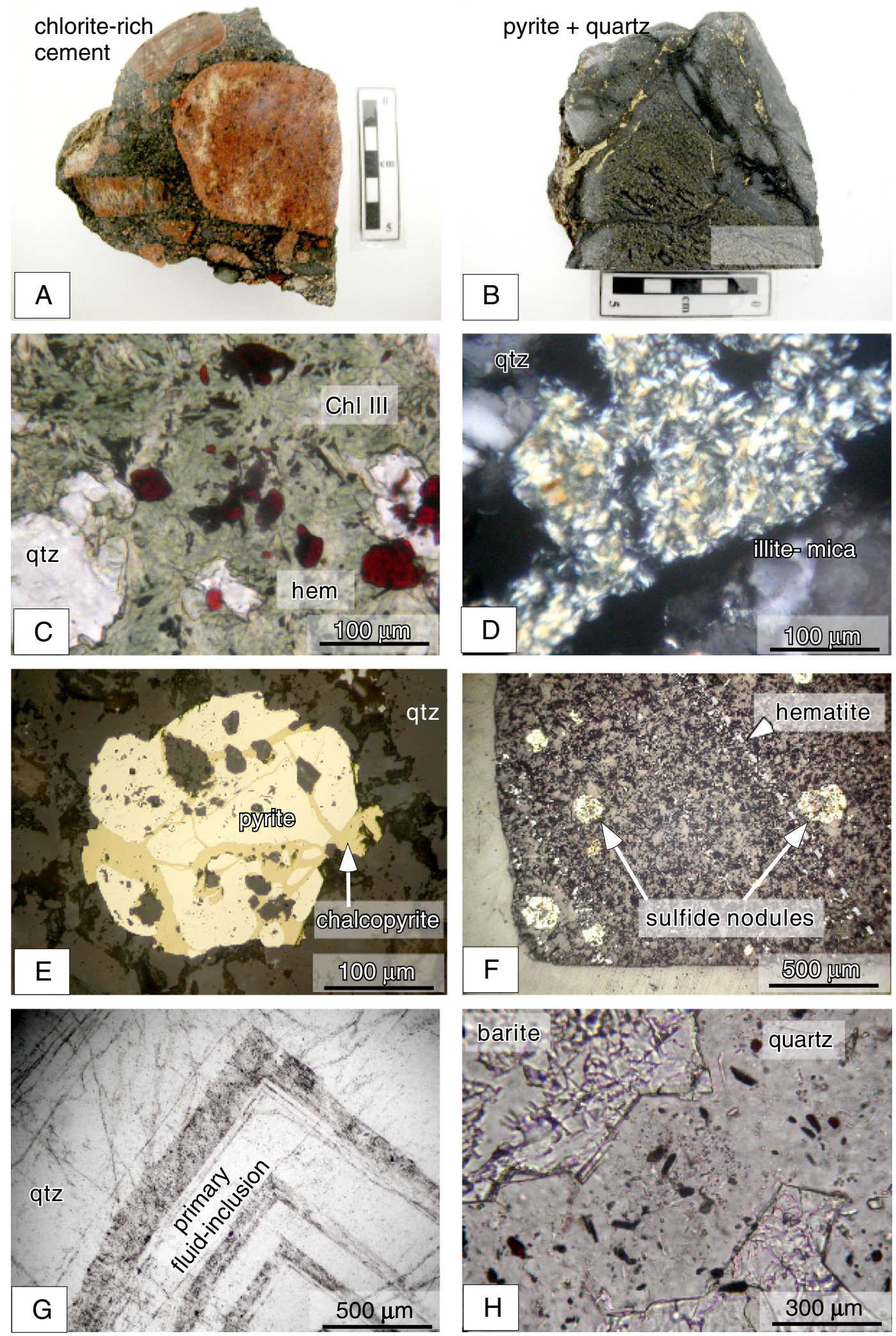

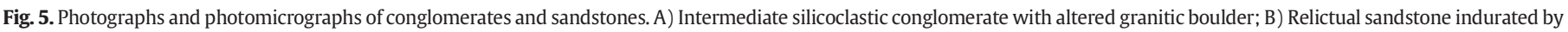

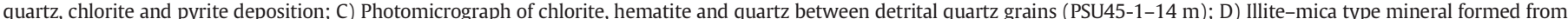

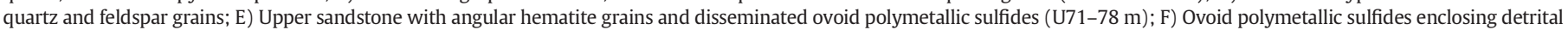

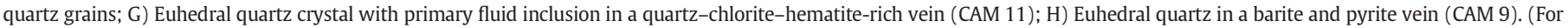
interpretation of the references to color in this figure, the reader is referred to the web version of this article.) 
Table 1

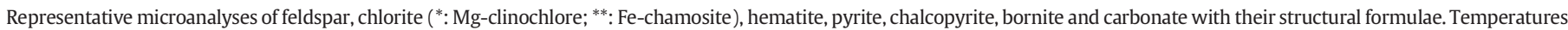
of chlorite formation (Inoue et al., 2010) were calculated for 10,15 and $20 \%$ of $\mathrm{Fe}_{\text {tot }}$ as $\mathrm{Fe}^{3+}$.

\begin{tabular}{|c|c|c|c|c|c|c|c|c|c|c|c|c|}
\hline & $\begin{array}{l}\text { K felds } \\
(\mathrm{n}=8)\end{array}$ & $\begin{array}{l}\text { plge } \\
(\mathrm{n}=15)\end{array}$ & $\begin{array}{l}\text { PTG } \\
\text { mica-illite }\end{array}$ & $\begin{array}{l}\text { PT49 } \\
(\mathrm{n}=23)^{*}\end{array}$ & $\begin{array}{l}\text { PT58 } \\
(\mathrm{n}=16)^{*}\end{array}$ & $\begin{array}{l}\text { PSU-45- } \\
1-14 \mathrm{~m} \\
(\mathrm{n}=5)^{*}\end{array}$ & $\begin{array}{l}\text { CAM } 1 \\
(\mathrm{n}=8) *\end{array}$ & $\begin{array}{l}\text { CAM } 3 \\
(\mathrm{n}=11)^{*}\end{array}$ & $\begin{array}{l}\text { CAM6 } \\
(\mathrm{n}=4)^{* *}\end{array}$ & $\begin{array}{l}\text { CAM10 } \\
(\mathrm{n}=10) \\
* *\end{array}$ & $\begin{array}{l}\text { CAM } 11 \\
(\mathrm{n}=13)^{*} \\
\text { and } * *\end{array}$ & $\begin{array}{l}\text { CAM } 12 \\
(\mathrm{n}=23)^{*} \\
\text { and }{ }^{* *}\end{array}$ \\
\hline $\mathrm{SiO}_{2}$ & $\begin{array}{l}61.10 \pm \\
0.48\end{array}$ & $\begin{array}{l}65.51 \pm \\
0.46\end{array}$ & $\begin{array}{l}49.18 \pm \\
2.48\end{array}$ & $\begin{array}{l}27.47 \pm \\
1.42\end{array}$ & $\begin{array}{l}28.45 \pm \\
2.20\end{array}$ & $29.79 \pm 2$ & $\begin{array}{l}25.00 \pm \\
0.60\end{array}$ & $\begin{array}{l}26.99 \pm \\
1.72\end{array}$ & $\begin{array}{l}24.90 \pm \\
1.78\end{array}$ & $\begin{array}{l}25.81 \pm \\
0.96\end{array}$ & $\begin{array}{l}25.70 \pm \\
1.42\end{array}$ & $\begin{array}{l}25.21 \pm \\
1.15\end{array}$ \\
\hline $\mathrm{TiO}_{2}$ & $\begin{array}{l}0.28 \pm \\
0.10\end{array}$ & $\begin{array}{l}0.01 \pm \\
0.01\end{array}$ & $\begin{array}{l}0.07 \pm \\
0.16\end{array}$ & $\begin{array}{l}0.02 \pm \\
0.02\end{array}$ & $\begin{array}{l}0.65 \pm \\
2.40\end{array}$ & $\begin{array}{l}0.21 \pm \\
0.5\end{array}$ & $\begin{array}{l}0.03 \pm \\
0.03\end{array}$ & $\begin{array}{l}0.05 \pm \\
0.01\end{array}$ & b.d.l. & $\begin{array}{l}0.05 \pm \\
0.01\end{array}$ & $\begin{array}{l}0.14 \pm \\
0.19\end{array}$ & $\begin{array}{l}0.04 \pm \\
0.03\end{array}$ \\
\hline $\mathrm{AI}_{2} \mathrm{O}_{3}$ & $\begin{array}{l}18.23 \pm \\
0.20\end{array}$ & $\begin{array}{l}19.16 \pm \\
0.13\end{array}$ & $\begin{array}{l}29.83 \pm \\
1.80\end{array}$ & $\begin{array}{l}18.88 \pm \\
1.74\end{array}$ & $\begin{array}{l}19.27 \pm \\
1.20\end{array}$ & $\begin{array}{l}21.36 \pm \\
0.82\end{array}$ & $\begin{array}{l}19.21 \pm \\
0.60\end{array}$ & $\begin{array}{l}19.46 \pm \\
1.13\end{array}$ & $\begin{array}{l}18.49 \pm \\
1.25\end{array}$ & $\begin{array}{l}19.07 \pm \\
0.85\end{array}$ & $\begin{array}{l}18.38 \pm \\
0.74\end{array}$ & $\begin{array}{l}18.36 \pm \\
0.65\end{array}$ \\
\hline $\mathrm{FeO}$ & $\begin{array}{l}0.29 \pm \\
0.08\end{array}$ & $\begin{array}{l}0.18 \pm \\
0.04\end{array}$ & $\begin{array}{l}2.30 \pm \\
0.84\end{array}$ & $\begin{array}{l}21.61 \pm \\
4.04\end{array}$ & $\begin{array}{l}19.97 \pm \\
2.15\end{array}$ & $\begin{array}{l}18.18 \pm \\
1.10\end{array}$ & $\begin{array}{l}27.16 \pm \\
1.44\end{array}$ & $\begin{array}{l}25.69 \pm \\
2.04\end{array}$ & $\begin{array}{l}34.90 \pm \\
1.89\end{array}$ & $\begin{array}{l}34.51 \pm \\
1.16\end{array}$ & $\begin{array}{l}30.49 \pm \\
0.90\end{array}$ & $\begin{array}{l}28.76 \pm \\
2.60\end{array}$ \\
\hline $\mathrm{MnO}$ & $\begin{array}{l}0.02 \pm \\
0.02\end{array}$ & $\begin{array}{l}0.01 \pm \\
0.02\end{array}$ & $\begin{array}{l}0.01 \pm \\
0.03\end{array}$ & $\begin{array}{l}0.07 \pm \\
0.03\end{array}$ & $\begin{array}{l}0.08 \pm \\
0.03\end{array}$ & $\begin{array}{l}0.56 \pm \\
0.06\end{array}$ & $\begin{array}{l}0.24 \pm \\
0.10\end{array}$ & $\begin{array}{l}0.25 \pm \\
0.07\end{array}$ & $\begin{array}{l}0.09 \pm \\
0.04\end{array}$ & $\begin{array}{l}0.085 \pm \\
0.04\end{array}$ & $\begin{array}{l}0.54 \pm \\
0.18\end{array}$ & $\begin{array}{l}0.48 \pm \\
0.21\end{array}$ \\
\hline $\mathrm{MgO}$ & $\begin{array}{l}0.16 \pm \\
0.02\end{array}$ & $\begin{array}{l}0.11 \pm \\
0.02\end{array}$ & $\begin{array}{l}1.78 \pm \\
0.37\end{array}$ & $\begin{array}{l}16.04 \pm \\
3.63\end{array}$ & $\begin{array}{l}16.59 \pm \\
1.98\end{array}$ & $\begin{array}{l}16.41 \pm \\
0.56\end{array}$ & $\begin{array}{l}11.87 \pm \\
0.89\end{array}$ & $\begin{array}{l}12.95 \pm \\
0.98\end{array}$ & $\begin{array}{l}7.77 \pm \\
0.93\end{array}$ & $\begin{array}{l}7.45 \pm \\
1.02\end{array}$ & $\begin{array}{l}9.94 \pm \\
0.73\end{array}$ & $\begin{array}{l}10.38 \pm \\
2.14\end{array}$ \\
\hline $\mathrm{CaO}$ & $\begin{array}{l}0.10 \pm \\
0.03\end{array}$ & $\begin{array}{l}0.07 \pm \\
0.06\end{array}$ & $\begin{array}{l}0.30 \pm \\
0.08\end{array}$ & $\begin{array}{l}0.13 \pm \\
0.05\end{array}$ & $\begin{array}{l}0.09 \pm \\
0.03\end{array}$ & $\begin{array}{l}0.23 \pm \\
0.01\end{array}$ & $\begin{array}{l}0.05 \pm \\
0.02\end{array}$ & $\begin{array}{l}0.07 \pm \\
0.03\end{array}$ & $\begin{array}{l}1.24 \pm \\
2.34\end{array}$ & $\begin{array}{l}0.11 \pm \\
0.06\end{array}$ & $\begin{array}{l}0.07 \pm \\
0.02\end{array}$ & $\begin{array}{l}0.03 \pm \\
0.02\end{array}$ \\
\hline $\mathrm{Na}_{2} \mathrm{O}$ & $\begin{array}{l}0.55 \pm \\
0.1\end{array}$ & $\begin{array}{l}11.31 \pm \\
0.14\end{array}$ & $\begin{array}{l}0.26 \pm \\
0.10\end{array}$ & $\begin{array}{l}0.10 \pm \\
0.02\end{array}$ & $\begin{array}{l}0.12 \pm \\
0.03\end{array}$ & 0.01 & $\begin{array}{l}0.28 \pm \\
0.05\end{array}$ & $\begin{array}{l}0.24 \pm \\
0.20\end{array}$ & b.d.l. & b.d.l. & $\begin{array}{l}0.11 \pm \\
0.02\end{array}$ & $\begin{array}{l}0.21 \pm \\
0.05\end{array}$ \\
\hline $\mathrm{K}_{2} \mathrm{O}$ & $\begin{array}{l}15.0 \pm \\
0.26\end{array}$ & $\begin{array}{l}0.07 \pm \\
0.03\end{array}$ & $\begin{array}{l}9.11 \pm \\
0.49\end{array}$ & $\begin{array}{l}0.05 \pm \\
0.08\end{array}$ & $\begin{array}{l}0.29 \pm \\
0.82\end{array}$ & $\begin{array}{l}0.05 \pm \\
0.05\end{array}$ & $\begin{array}{l}0.14 \pm \\
0.05\end{array}$ & $\begin{array}{l}0.29 \pm \\
0.18\end{array}$ & $\begin{array}{l}0.18 \pm \\
0.03\end{array}$ & $\begin{array}{l}0.37 \pm \\
0.29\end{array}$ & $\begin{array}{l}0.11 \pm \\
0.05\end{array}$ & $\begin{array}{l}0.15 \pm \\
0.20\end{array}$ \\
\hline Total & $\begin{array}{l}95.74 \pm \\
0.60\end{array}$ & $\begin{array}{l}96.42 \pm \\
0.65\end{array}$ & $\begin{array}{l}92.85 \pm \\
2.60\end{array}$ & $\begin{array}{l}84.35 \pm \\
1.91\end{array}$ & $\begin{array}{l}85.51 \pm \\
1.75\end{array}$ & $\begin{array}{l}86.1 \pm \\
0.1\end{array}$ & $\begin{array}{l}83.98 \pm \\
1.49\end{array}$ & $\begin{array}{l}85.99 \pm \\
1.37\end{array}$ & $\begin{array}{l}87.60 \pm \\
1.60\end{array}$ & $\begin{array}{l}87.45 \pm \\
1.61\end{array}$ & $\begin{array}{l}85.49 \pm \\
1.14\end{array}$ & $\begin{array}{l}83.63 \pm \\
0.80\end{array}$ \\
\hline 0 & 32 & 32 & 11 & 28 & 28 & 28 & 28 & 28 & 28 & 28 & 28 & 28 \\
\hline $\mathrm{Si} / \mathrm{Si}(\mathrm{T} 1 \mathrm{~T} 2)$ & 11.86 & 11.90 & 3.32 & 5.83 & 5.94 & 6.01 & 5.53 & 5.75 & 5.49 & 5.65 & 5.67 & 5.65 \\
\hline $\mathrm{Al}^{\mathrm{IV}}, \mathrm{Al}^{\mathrm{IV}(\mathrm{T} 2)}$ & 4.17 & 4.10 & 2.37 & 2.17 & 2.06 & 1.99 & 2.48 & 2.25 & 2.51 & 2.35 & 2.33 & 2.35 \\
\hline $\mathrm{Al}^{\mathrm{VI}(\mathrm{M} 1)}$ & - & - & - & 2.55 & 2.68 & 3.08 & 2.53 & 2.63 & 2.30 & 2.57 & 2.46 & 2.50 \\
\hline $\mathrm{Ti}$ & - & - & 0.00 & 0.004 & 0.10 & 0.03 & 0.01 & 0.01 & - & 0.01 & 0.02 & 0.01 \\
\hline $2 \mathrm{Fe}^{+3}$ & - & - & 0.13 & $\begin{array}{l}0.58 \\
\left(\mathrm{Fe}^{3+}:\right. \\
15 \%)\end{array}$ & $\begin{array}{l}0.52 \\
\left(\mathrm{Fe}^{3+}:\right. \\
15 \%)\end{array}$ & $\begin{array}{l}0.46 \\
\left(\mathrm{Fe}^{3+}:\right. \\
15 \%)\end{array}$ & $\begin{array}{l}0.75 \\
\left(\mathrm{Fe}^{3+}:\right. \\
15 \%)\end{array}$ & $\begin{array}{l}0.68 \\
\left(\mathrm{Fe}^{3+}:\right. \\
15 \%)\end{array}$ & $\begin{array}{l}0.97 \\
\left(\mathrm{Fe}^{3+}:\right. \\
15 \%)\end{array}$ & $\begin{array}{l}0.95 \\
\left(\mathrm{Fe}^{3+}:\right. \\
15 \%)\end{array}$ & $\begin{array}{l}0.84 \\
\left(\mathrm{Fe}^{3+}:\right. \\
15 \%)\end{array}$ & $\begin{array}{l}0.81 \\
\left(\mathrm{Fe}^{3+}:\right. \\
15 \%)\end{array}$ \\
\hline $2 \mathrm{Fe}^{+2}$ & 0.10 & 0.03 & 0.00 & 3.26 & 2.96 & 2.605 & 4.27 & 3.89 & 5.47 & 5.37 & 4.78 & 4.58 \\
\hline Mn & - & - & 0.00 & 0.01 & 0.01 & 0.1 & 0.05 & 0.05 & 0.02 & 0.02 & 0.10 & 0.09 \\
\hline $\mathrm{Mg}$ & - & - & 0.18 & 5.07 & 5.16 & 4.93 & 3.91 & 4.11 & 2.55 & 2.43 & 3.27 & 3.47 \\
\hline $\mathrm{Ca}$ & 0.02 & 0.01 & 0.02 & 0.03 & 0.02 & 0.05 & 0.01 & 0.02 & 0.29 & 0.03 & 0.02 & 0.01 \\
\hline $\mathrm{Na}$ & 0.21 & 3.98 & 0.03 & 0.04 & 0.05 & 0 & 0.12 & 0.10 & & & 0.05 & 0.09 \\
\hline K & 3.71 & 0.02 & 0.78 & 0.01 & 0.08 & 0.01 & 0.04 & 0.08 & 0.05 & 0.10 & 0.03 & 0.04 \\
\hline $\begin{array}{l}\mathrm{H} / \mathrm{OH}^{*} \\
\quad \text { (chlorite) }\end{array}$ & - & - & 2.00 & 16 & 16 & 16 & 16 & 16 & 16 & 16 & 16 & 16 \\
\hline $\begin{array}{c}\text { Number of } \\
\text { vacant }\end{array}$ & & & & 0.53 & 0.66 & 0.83 & 0.49 & 0.64 & 0.70 & 0.66 & 0.54 & 0.552 \\
\hline An & 0.5 & 0.3 & $\begin{array}{l}\text { Inoue et al. } \\
\text { (2010) }\end{array}$ & $\begin{array}{l}\mathrm{Fe}^{3+} 20 \%: \\
225{ }^{\circ} \mathrm{C}\end{array}$ & $\begin{array}{l}\mathrm{Fe}^{3+} 20 \%: \\
183{ }^{\circ} \mathrm{C}\end{array}$ & $\begin{array}{l}\mathrm{Fe}^{3+} 20 \%: \\
139{ }^{\circ} \mathrm{C}\end{array}$ & $\begin{array}{l}\mathrm{Fe}^{3+} 20 \%: \\
280{ }^{\circ} \mathrm{C}\end{array}$ & $\begin{array}{l}\mathrm{Fe}^{3+} 20 \%: \\
215^{\circ} \mathrm{C}\end{array}$ & $\begin{array}{l}\mathrm{Fe}^{3+} 20 \%: \\
262{ }^{\circ} \mathrm{C}\end{array}$ & $\begin{array}{l}\mathrm{Fe}^{3+} 20 \%: \\
226{ }^{\circ} \mathrm{C}\end{array}$ & $\begin{array}{l}\mathrm{Fe}^{3+} 20 \%: \\
248{ }^{\circ} \mathrm{C}\end{array}$ & $\begin{array}{l}\mathrm{Fe}^{3+} 20 \%: \\
260{ }^{\circ} \mathrm{C}\end{array}$ \\
\hline $\mathrm{Ab}$ & 5.2 & 99.3 & & $\begin{array}{l}\mathrm{Fe}^{3+} 15 \%: \\
233{ }^{\circ} \mathrm{C}\end{array}$ & $\begin{array}{l}\mathrm{Fe}^{3+} 15 \%: \\
188{ }^{\circ} \mathrm{C}\end{array}$ & $\begin{array}{l}\mathrm{Fe}^{3+} 15 \%: \\
142{ }^{\circ} \mathrm{C}\end{array}$ & $\begin{array}{l}\mathrm{Fe}^{3+} 15 \%: \\
293{ }^{\circ} \mathrm{C}\end{array}$ & $\begin{array}{l}\mathrm{Fe}^{3+} 15 \%: \\
222{ }^{\circ} \mathrm{C}\end{array}$ & $\begin{array}{l}\mathrm{Fe}^{3+} 15 \%: \\
270{ }^{\circ} \mathrm{C}\end{array}$ & $\begin{array}{l}\mathrm{Fe}^{3+} 15 \%: \\
236{ }^{\circ} \mathrm{C}\end{array}$ & $\begin{array}{l}\mathrm{Fe}^{3+} 15 \%: \\
260{ }^{\circ} \mathrm{C}\end{array}$ & $\begin{array}{l}\mathrm{Fe}^{3+} 15 \%: \\
275{ }^{\circ} \mathrm{C}\end{array}$ \\
\hline Or & 94.3 & 0.4 & & $\begin{array}{l}\mathrm{Fe}^{3+} 10 \%: \\
244{ }^{\circ} \mathrm{C}\end{array}$ & $\begin{array}{l}\mathrm{Fe}^{3+} 10 \%: \\
195{ }^{\circ} \mathrm{C}\end{array}$ & $\begin{array}{l}\mathrm{Fe}^{3+} 10 \%: \\
145^{\circ} \mathrm{C}\end{array}$ & $\begin{array}{l}\mathrm{Fe}^{3+} 10 \%: \\
310^{\circ} \mathrm{C}\end{array}$ & $\begin{array}{l}\mathrm{Fe}^{3+} 10 \%: \\
231^{\circ} \mathrm{C}\end{array}$ & $\begin{array}{l}\mathrm{Fe}^{3+} 10 \%: \\
282{ }^{\circ} \mathrm{C}\end{array}$ & $\begin{array}{l}\mathrm{Fe}^{3+} 10 \%: \\
248{ }^{\circ} \mathrm{C}\end{array}$ & $\begin{array}{l}\mathrm{Fe}^{3+} 10 \%: \\
276{ }^{\circ} \mathrm{C}\end{array}$ & $\begin{array}{l}\mathrm{Fe}^{3+} 10 \%: \\
310{ }^{\circ} \mathrm{C}\end{array}$ \\
\hline Sulfides & \multicolumn{3}{|c|}{ Pyrite $(\mathrm{n}=13)$} & \multicolumn{3}{|c|}{$\mathrm{Ccp}(\mathrm{n}=19)$} & \multicolumn{2}{|l|}{ Bor $(n=25)$} & \multicolumn{2}{|c|}{$\mathrm{Cc}(\mathrm{n}=5)$} & \multicolumn{2}{|r|}{$\operatorname{Hem}(n=6)$} \\
\hline S & \multicolumn{3}{|c|}{$52.62 \pm 0.24$} & \multicolumn{3}{|c|}{$34.85 \pm 0.33$} & \multicolumn{2}{|l|}{$25.93 \pm 0.43$} & \multicolumn{2}{|c|}{$20.20 \pm 0.24$} & \multicolumn{2}{|r|}{$0.15 \pm 0.04$} \\
\hline $\mathrm{Fe}$ & & \multicolumn{2}{|c|}{$46.22 \pm 0.39$} & \multicolumn{3}{|c|}{$30.56 \pm 0.44$} & \multicolumn{2}{|l|}{$11.40 \pm 0.58$} & \multicolumn{2}{|c|}{$0.10 \pm 0.06$} & \multicolumn{2}{|r|}{$98.49 \pm 1.30$} \\
\hline $\mathrm{Cu}$ & & \multicolumn{2}{|c|}{$0.58 \pm 0.29$} & \multicolumn{3}{|c|}{$34.31 \pm 0.42$} & $62.38 \pm 0.90$ & & $79.39=$ & 0.34 & & $0.93 \pm 0.33$ \\
\hline $\mathrm{Ni}$ & & $0.04 \pm 0.04$ & & $0.02 \pm$ & $=0.03$ & & $0.03 \pm 0.04$ & & $0.03 \pm$ & 0.04 & & $0.02 \pm 0.02$ \\
\hline Co & & $0.17 \pm 0.15$ & & $0.08 \pm$ & $=0.13$ & & $0.07 \pm 0.09$ & & $0.06 \pm$ & 0.06 & & $0.14 \pm 0.04$ \\
\hline $\mathrm{Pb}$ & & $0.31 \pm 0.23$ & & $0.13 \pm$ & $=0.16$ & & $0.14 \pm 0.14$ & & $0.18 \pm$ & 0.15 & & $0.15 \pm 0.09$ \\
\hline $\mathrm{Zn}$ & & $0.04 \pm 0.04$ & & $0.01 \pm$ & $=0.05$ & & b.d.l. & & b.d.l. & & & $0.08 \pm 0.06$ \\
\hline As & & $0.01 \pm 0.03$ & & $0.02 \pm$ & $=0.02$ & & $0.03 \pm 0.04$ & & $0.02 \pm$ & 0.03 & & $0.03 \pm 0.03$ \\
\hline $\mathrm{Au}$ & & b.d.l. & & $0.01 \pm$ & $=0.02$ & & $0.01 \pm 0.03$ & & $0.01 \pm$ & 0.01 & & $0.01 \pm 0.01$ \\
\hline S (apfu) & & 0.661 & & 0.544 & & & 0.405 & & 0.335 & & & \\
\hline $\mathrm{Fe}$ & & 0.333 & & 0.184 & & & 0.102 & & 0.001 & & & \\
\hline $\mathrm{Cu}$ & & 0.004 & & 0.270 & & & 0.492 & & 0.663 & & & \\
\hline $\mathrm{Ni}$ & & 2.7E-04 & & $1.7 \mathrm{E}-0$ & & & $2.6 \mathrm{E}-04$ & & $2.7 \mathrm{E}-0$ & & & \\
\hline Co & & $1.2 \mathrm{E}-03$ & & $6.8 \mathrm{E}-0$ & & & $5.9 \mathrm{E}-04$ & & $5.4 \mathrm{E}-0$ & & & \\
\hline $\mathrm{Pb}$ & & $6.0 \mathrm{E}-04$ & & $3.1 \mathrm{E}-0$ & & & $3.4 \mathrm{E}-04$ & & $4.6 \mathrm{E}-0$ & & & \\
\hline $\mathrm{Zn}$ & & $2.5 \mathrm{E}-04$ & & 7.7E-0 & & & - & & - & & & \\
\hline $\mathrm{Au}$ & & - & & $2.5 \mathrm{E}-0$ & & & $2.5 \mathrm{E}-05$ & & $2.7 \mathrm{E}-0$ & & & \\
\hline Carbonates & & & $\begin{array}{l}\text { CAM } 3 \\
(n=13)\end{array}$ & & & and max & & & $\begin{array}{l}\text { CAM } 12 \\
(n=15)\end{array}$ & & & Min and max \\
\hline $2 \mathrm{Fe}^{+2}$ & & & $0.58 \pm 0.53$ & & & 4 to 1.27 & & & $0.51 \pm 0.47$ & & & 0.06 to 1.47 \\
\hline $\mathrm{Mn}$ & & & $0.08 \pm 0.75$ & & & 4 to 0.03 & & & $0.09 \pm 0.03$ & & & 0.09 to 0.03 \\
\hline $\mathrm{Mg}$ & & & $0.78 \pm 0.75$ & & & 4 to 1.78 & & & $0.54 \pm 0.48$ & & & 0.03 to 1.26 \\
\hline $\mathrm{Ca}$ & & & $4.55 \pm 1.24$ & & & 7 to 2.92 & & & $4.85 \pm 0.92$ & & & 5.83 to 3.23 \\
\hline
\end{tabular}


Table 2

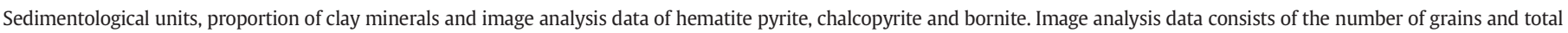
surface proportion of opaques as pyrite, chalcopyrite, bornite and hematite.

\begin{tabular}{|c|c|c|c|c|c|c|c|c|c|c|c|c|}
\hline & $\begin{array}{l}\text { Drill core } \\
\text { (name) }\end{array}$ & $\begin{array}{l}\text { Sample depth } \\
(\mathrm{m})\end{array}$ & $\begin{array}{l}\text { Weight \% particle } \\
<10 \mu \mathrm{m} \text { fraction* }\end{array}$ & $\begin{array}{l}\text { Weight \% } \\
\text { opaque }\end{array}$ & $\begin{array}{l}\text { Number of } \\
\text { opaque grain }\end{array}$ & $\begin{array}{l}\% \text { sulfide/ } \\
\text { thin section }\end{array}$ & Average size & $\begin{array}{l}\text { StDev } \\
\left(\mathrm{mm}^{2}\right)\end{array}$ & $\begin{array}{l}\text { Surface } \% \\
\text { of pyrite }\end{array}$ & $\begin{array}{l}\text { Surface \% of } \\
\text { chalcopyrite }\end{array}$ & $\begin{array}{l}\text { Surface } \% \\
\text { of bornite }\end{array}$ & $\begin{array}{l}\text { Surface \% } \\
\text { of hematite }\end{array}$ \\
\hline \multirow{8}{*}{$\begin{array}{l}\text { Upper } \\
\text { conglomerate }\end{array}$} & \multirow[t]{8}{*}{ U71 } & 28.3 & 7 & & 227 & 0.060 & 0.022 & 0.018 & Trace & & & 100 \\
\hline & & 49.1 & 10 & & 1227 & 0.360 & 0.011 & 0.011 & Trace & & & 100 \\
\hline & & 72.5 & 15.0 & & n.d. & & & & & & & \\
\hline & & 75.4 & 14.0 & & n.d. & & & & & & & \\
\hline & & 76.5 & 13.6 & & n.d. & & & & & & & \\
\hline & & 83.3 & 14.1 & & 1895 & 0.140 & 0.012 & 0.012 & Trace & & & 100 \\
\hline & & 93.0 & 15.3 & & 453 & 0.220 & 0.015 & 0.015 & Trace & Trace & & 100 \\
\hline & & 95.0 & 12.0 & & n.d. & & & & & & & \\
\hline \multirow{3}{*}{$\begin{array}{l}\text { Intermediate } \\
\text { sandstone }\end{array}$} & \multirow[t]{3}{*}{ U71 } & 35.2 & 10.2 & & & & & & & & & \\
\hline & & 59.0 & 8.4 & & & & & & & & & \\
\hline & & 68.5 & 7.1 & & & & & & & & & \\
\hline \multirow{10}{*}{$\begin{array}{l}\text { Upper } \\
\text { sandstone }\end{array}$} & \multirow[t]{8}{*}{ PSU45 } & 34.8 & 11.2 & & 40 & 0.744 & 0.070 & 0.069 & 88.71 & 11.29 & & \\
\hline & & 5.90 & 10.7 & & 35 & 0.104 & 0.011 & 0.022 & 100.00 & & & \\
\hline & & 61.8 & 13.0 & & & & & & & & & \\
\hline & & 64.0 & 14.2 & & & & & & & & & \\
\hline & & 70.0 & 10.1 & & 164 & 1.511 & 0.034 & 0.037 & 52.60 & 15.90 & 31.00 & 0.41 \\
\hline & & 71.0 & 9.7 & & 1446 & 1.190 & 0.012 & 0.011 & 59.90 & 31.84 & 7.56 & 0.56 \\
\hline & & 75.0 & 8.9 & & 101 & 1.102 & 0.045 & 0.129 & 57.40 & 40.10 & 2.46 & \\
\hline & & 78.0 & 11.1 & & 243 & 1.626 & 0.025 & 0.023 & 75.44 & 16.25 & 8.30 & \\
\hline & \multirow[t]{2}{*}{ U180 } & 59.0 & 5.5 & & 1114 & 1.060 & 0.212 & 0.050 & 92.00 & 8.00 & & \\
\hline & & 64.7 & 7.4 & & 2478 & 1.770 & 0.179 & 0.054 & 64.83 & 33.50 & & 1.67 \\
\hline
\end{tabular}
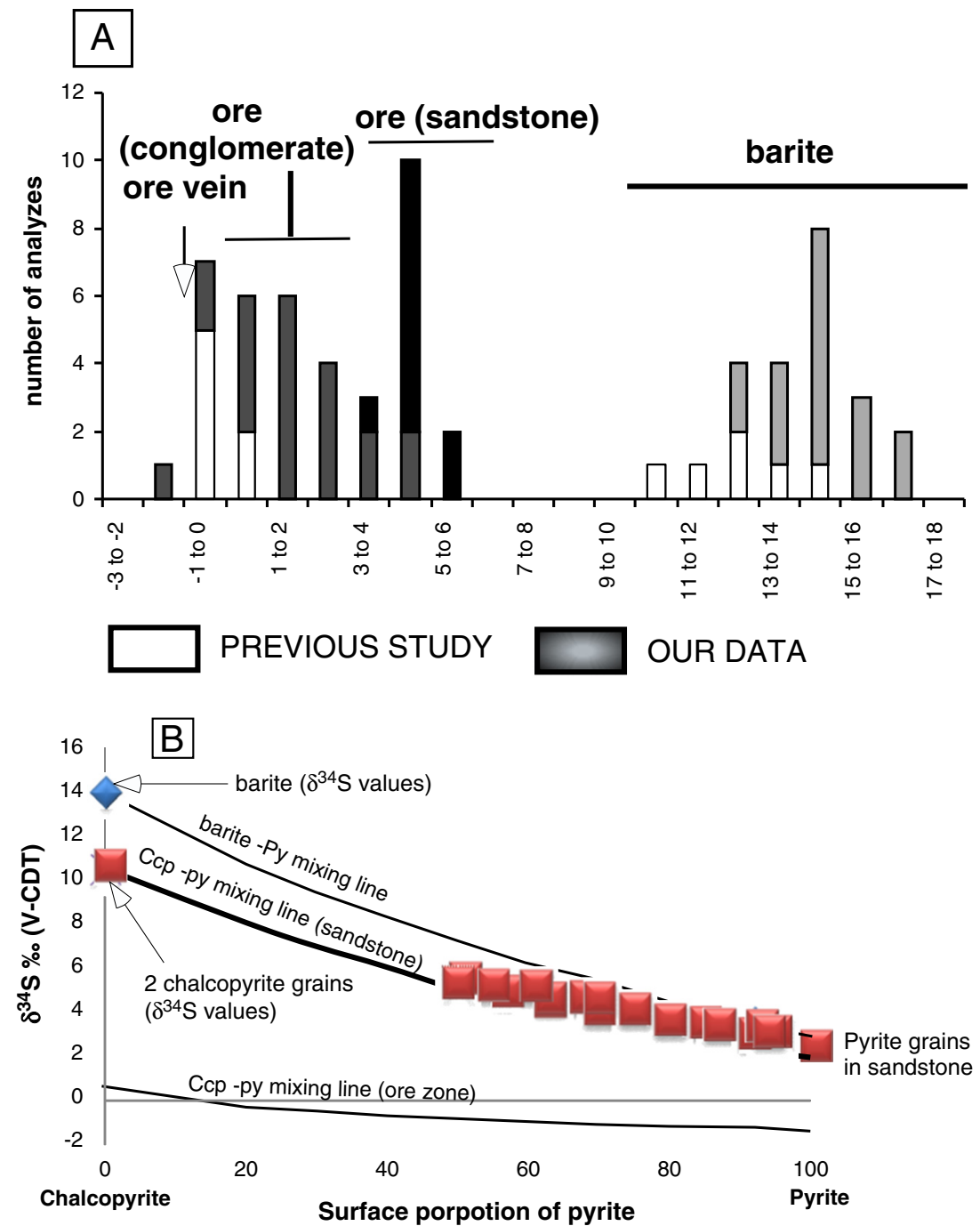

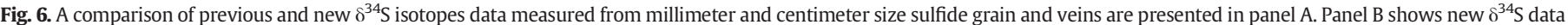

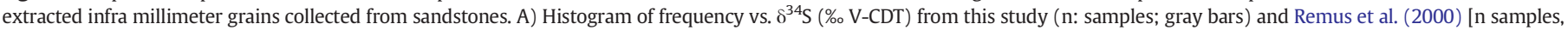

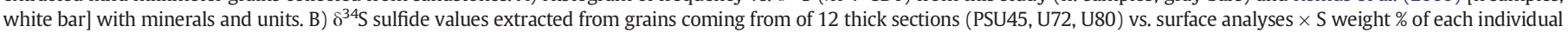
grain analyzed (Table 1). For comparison, mixing trends are plotted using bibliographical values (Remus et al., 2000). 
Mg-clinochlore in cemented sandstone (IIb polytype; PT-49) and Fe-chamosite (Ib90 ${ }^{\circ}$ polytype; CAM 12 ) that fill vugs).

\subsection{Ore petrography and chemical composition}

The disseminated sulfide ores occupy sandstones and sulfide grain includes angulose quartz and feldspar grains. Optical and electron microscopic observations allow recognition of pyrite (Py), chalcopyrite $(\mathrm{Ccp})$, bornite $(\mathrm{Bn})$ and chalcocite $(\mathrm{Cc})$ assemblages. There is no exsolution texture in $\mu \mathrm{m}$ to $\mathrm{cm}$ size sulfides, but rather, fractures and replacements of pyrite by chalcopyrite and pyrite-chalcopyrite grains by bornite, or scarce pyrite grains totally replaced by bornite (SEM observation). Gentle sandstone disaggregation allowed the collection of the clay-micas-chlorite-rich size fractions, angulose hematite and ovoid sulfides from each rock sample analyzed (Fig. 6). An estimate of clays, hematite, sulfides and other silicates was then obtained by their relative weight proportion (weight \%; Table 1). Clay materials are dominant compared to hematite and sulfides grains, except in upper conglomerate unit where hematite grains dominate compared to scarce sulfides. In the sandstones sulfides are up to $1.8 \%$ of a thin section (Table 2) with a dominant proportion of pyrite compared to chalcopyrite, bornite and chalcocite. Pyrite grains have broken cubic to quadratic shapes, whereas polymetallic sulfides (Py $+\mathrm{Ccp}+\mathrm{Bn}+\mathrm{Cc}$ ) grains have ovoid shapes. In thin section, cubic to ovoid sulfide grains enclose small detrital disseminated quartz grains (Fig. 5F), angulose quartz and sulfides being surrounded by quartz overgrowth and chlorite flakes. Ovoid sulfide grains and veins consist cubic to quadratic pyrite; chalcopyrite surrounds or fills broken pyrite. Bornite is found in outer rims of sulfide grains (chalcopyrite and pyrite). Electron microprobe analyses suggest that sulfides in veins, disseminated in sandstone and barite bearing veins have similar element compositions (Table 1 ) to those referred by Ronchi et al. (2000). Trace metal contents in sulfides (Pb, $\mathrm{Zn}, \mathrm{Ni}$ and $\mathrm{Au}$ ) show an ambivalent Ni-enrichment, whereas $\mathrm{Co}, \mathrm{Pb}, \mathrm{Zn}$ are enriched in pyrite and $\mathrm{Au}$ associated in bornite. In sandstone units, petrographic observation showed a dominant number of sulfides with ovoid shapes. Image processing of sulfides observed in reflected light allowed the calculation of the proportion of each sulfide grain for hundreds of grains (Table 1). None of this image processing showed that the occurrence of chalcopyrite or bornite was dependent on depth, shape or crystal size (Table 1). This image processing allowed the selection of grains with different proportions of pyrite, chalcopyrite and bornite. Further polymetallic-sulfide grains were individually extracted and analyzed for stable sulfur isotopes.

Meter-wide barite veins were sampled to characterize S-rich oxidized fluids (Church and Wolgemuth, 1972; Hanor, 2000). Barite veins consist of cm-size crystals, some of which have a S-flexuous shape. Some large barite crystals host scarce euhedral pyrite and chalcopyrite crystals (Fig. 6 in Troian et al., 2009), euhedral quartz $(<\mathrm{mm})$ and dusty hematite $(\mu \mathrm{m})$.

Among conglomerate and sandstone units, carbonate precipitation occurs as lenses, veinlets ( $\mathrm{mm}$ size) or as intergranular cement $(<0.1 \mathrm{~mm}$ ) replacing quartz overgrowths, euhedral quartz and barite crystals. Electron microprobe analyses show Ca-dominant to $\mathrm{Ca}>\mathrm{Mg}-$ Fe carbonates (Table 1).

\section{3. $\mathrm{O}, \mathrm{H}, \mathrm{C}$ and $\mathrm{S}$ stable isotope values}

Specular hematite $\left(\delta^{18} \mathrm{O}=-1.9 \%\right.$ ) and euhedral quartz $\left(\delta^{18} \mathrm{O}=\right.$ $12.2 \%$ ) associated with Fe-chamosite (CAM $12<2 \mu \mathrm{m}$ : $\delta \mathrm{D}=-44 \%$ 。 and $\delta^{18} \mathrm{O}=9.8 \%$ ) in the residual ore zone (CAM 11; Fig. 4). The oxygen

Table 3

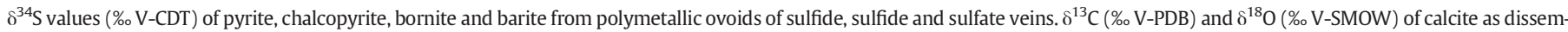
inated cement or lenses in sandstone and conglomerate units (NB. Same vein as those analyzed for fluid inclusion in Ronchi et al., 2000),

\begin{tabular}{|c|c|c|c|c|c|c|c|c|c|}
\hline & $\begin{array}{l}\text { Drill core } \\
\text { (name) }\end{array}$ & $\begin{array}{l}\text { Sample } \\
\text { depth } \\
(\mathrm{m})\end{array}$ & $\begin{array}{l}\delta^{34} \mathrm{~S} \% \\
(\mathrm{~V}-\mathrm{CDT}) \\
\text { Pyrite grain }\end{array}$ & $\begin{array}{l}\delta^{34} \mathrm{~S} \% \text { (V-CDT) } \\
\text { Chalcopyrite vein }\end{array}$ & $\begin{array}{l}\delta^{34} \mathrm{~S} \% \text { (V-CDT) } \\
\text { Mixed chalco-pyr- } \\
\text { bor grain }\end{array}$ & $\begin{array}{l}\delta^{34} \mathrm{~S} \% \\
(\mathrm{~V}-\mathrm{CDT}) \\
\text { Bornite vein }\end{array}$ & $\begin{array}{l}\delta^{34} \mathrm{~S} \% \\
(\mathrm{~V}-\mathrm{CDT}) \\
\text { Barite vein }\end{array}$ & $\begin{array}{l}\delta^{13} \mathrm{C} \% \\
\text { (V-PDB) } \\
\text { Calcite }\end{array}$ & $\begin{array}{l}\delta^{18} \mathrm{O} \% \\
(\mathrm{~V}-\mathrm{SMOW})\end{array}$ \\
\hline \multirow[t]{10}{*}{ Upper conglomerate } & U71 & 28.3 & 1.2 & & & & & & \\
\hline & & 49.1 & 1.1 & & & & & & \\
\hline & & 72.5 & & & & & & -1.85 & 17.3 \\
\hline & & 75.4 & 1.4 & & & & & -1.86 & 16.7 \\
\hline & & $75.4 \mathrm{~B}$ & & & & & & -1.71 & 18.1 \\
\hline & & $75.4 \mathrm{C}$ & & & & & & -1.92 & 6.6 \\
\hline & & 76.5 & & & & & 12.9 to $14.4(\mathrm{n}: 4)$ & -1.87 & 19.8 \\
\hline & & 83.3 & 1.1 & 1.7 to $2.5(\mathrm{n}=3)$ & & & & -2.09 & 17.8 \\
\hline & & 93.0 & 1.3 & 1.0 & 4.8 & & 16 and 16.3 & & \\
\hline & & 95.0 & & 0.8 & 3.4 & & 14.8 to $16(\mathrm{n}: 3)$ & & \\
\hline \multirow{3}{*}{$\begin{array}{c}\text { Intermediate } \\
\text { sandstone }\end{array}$} & U71 & 35.2 & & & & & & -2.53 & 17.5 \\
\hline & & 59.0 & & & 4.2 to $5.6(\mathrm{n}: 6)$ & & 15 and 15.2 & -2.65 & 5.8 \\
\hline & & 68.5 & & & & & & -3.03 & 16.9 \\
\hline \multirow[t]{8}{*}{ Upper sandstone } & PSU45 & 34.8 & -1.6 & & 3.2 to $3.4(\mathrm{n}: 6)$ & & & & \\
\hline & & 59.0 & & & & & 12 & & \\
\hline & & 61.8 & & & 4.1 to $5.3(n=4)$ & & 13.8 and 15.7 & & \\
\hline & & 64.0 & & 2.55 & & & & & \\
\hline & & 70.0 & 1.9 & 1.5 and 1.9 & 3.7 & 1.5 to $2.3(\mathrm{n}=3)$ & & & \\
\hline & & 71.0 & & & & & & & \\
\hline & & 75.0 & & 1.3 & & & 15.3 & & \\
\hline & & 78.0 & & & & & & & \\
\hline \multirow[t]{2}{*}{ Upper sandstone } & U180-94-72 & 59.0 & 1.7 & & $\begin{array}{l}4.4 \text { to } 5(\mathrm{n}: 4) ; 10.5 \\
(100 \% \text { chalco) }\end{array}$ & & & & \\
\hline & & 64.7 & & & 2 to $5.7(\mathrm{n}: 6)$ & & & & \\
\hline Up conglo & Outcrop & CAM 4 & & & & & 12.8 & & \\
\hline Up conglo & & CAM 5 & & & & & 13.7 and 14.5 & & \\
\hline Inter sands & & CAM 9 & 1.9 & & & & 13.7 and 14.1 & & \\
\hline Up conglo & & CAM6 & & -0.9 (ore zone) & & & 14.4 & & \\
\hline Up conglo & & CAM5 & 1.1 & & & & 13.7 & & \\
\hline Up conglo & & CAM 8 & & & & & & & \\
\hline Up conglo & & CAM 11 & & $\begin{array}{l}-0.8 \pm 0.3 \\
\text { (ore zone; } \mathrm{n}=5 \text { ) }\end{array}$ & & & & & \\
\hline Up conglo & & CAM 12 & & -1.8 (ore zone) & & & & & \\
\hline
\end{tabular}


Table 4

$\delta^{34} \mathrm{~S}$ values (\% V-CDT) of pyrite and chalcopyrite from polymetallic ovoids of sulfide and monomineral sulfides in terms of surface pyrite content (c.f. Table 3 ).

\begin{tabular}{llll}
\hline $\begin{array}{l}\text { Surface \% of pyrite } \\
\text { Py / (Py + Ccp) }\end{array}$ & $\begin{array}{l}\text { Mineral } \\
\text { Image analysis }\end{array}$ & $\begin{array}{l}\text { Weight \% of S } \\
\text { EA-C-IRMS }\end{array}$ & $\begin{array}{l}\delta^{34} \mathrm{~S} \% \text { (V-CDT) } \\
\text { Ovoid grain }\end{array}$ \\
\hline 95 & - & & \\
85 & qtz & 41 & 3.2 \\
87 & qtz & 47 & 3.6 \\
92 & - & 50.6 & 3.5 \\
93 & - & 50.7 & 3.1 \\
94 & - & 51 & 3.45 \\
80 & - & 48.4 & 3.2 \\
70 & qtz & 46.6 & 3.7 \\
75 & - & 47.5 & 4.2 \\
68 & - & 46.2 & 4.2 \\
63 & qtz & 42 & 4.75 \\
63 & - & 45.3 & 4.56 \\
57 & - & 44.2 & 4.7 \\
51 & qtz & 41 & 5 \\
50 & - & 43 & 5.6 \\
70 & qtz & 42 & 5.4 \\
61 & - & 45 & 4.7 \\
55 & qtz & 40 & 5.25 \\
\hline
\end{tabular}

and hydrogen values of chlorites were determined from two separations of pure Fe-Mg chamosite and Mg-clinochlore (PSU45-1-14 m < $2 \mu \mathrm{m}: \delta \mathrm{D}=-56 \% ; \delta^{18} \mathrm{O}=8.4 \%$; PT- $49<2 \mu \mathrm{m}: \delta \mathrm{D}=-47 \%$ 。 $\delta^{18} \mathrm{O}=8.5 \%$ ) to complete the previous $\mathrm{O}$ and $\mathrm{H}$ stable isotope data.

In the residual ore zone, located in the lower part of the Uruguay open pit, two decimeter-wide veins are filled by pyrite and chalcopyrite. New $\delta^{34} \mathrm{~S}$ data (Table 3 ) in 1.0 to $2.5 \mathrm{~cm}$-wide veins of pyrite and chalcopyrite are similar to those presented by Remus et al. (2000). Nevertheless, the new $\delta^{34} \mathrm{~S}$ values (60 analyses; Tables 3 and 4 ) of pyrite, chalcopyrite and bornite in veins or the porosity of sedimentary units are more scattered and vary from 6 to $-1 \%$ (Fig. $6 \mathrm{~A}$ ). $\delta^{34} \mathrm{~S}$ values measured in disseminated ovoid-shaped and polymetallic sulfides from different levels are enriched in ${ }^{34} \mathrm{~S}$ isotopes compared to large ore veins (Fig. 6A). Eighteen ovoid sulfide grains from 3 thin sections with different proportions of Py $+\mathrm{Ccp}$ previously identified by image analysis (Table 2 ) were picked up from thick sections and analyzed for $\delta^{34} \mathrm{~S}$ values (Table 4). Surface proportions of pyrite or chalcopyrite (image analysis), weight $\%$ of sulfur and $\delta^{34} \mathrm{~S}$ values (EA-C-IRMS) form a trend line (Fig. 6B, Table 4). The trend line of the measured weight $\%$ of $\mathrm{S}$ and calculated surface proportion \% using image analyses versus $\delta^{34} \mathrm{~S}$ are different. Some low $\mathrm{S}$ contents determined with EA-C-IRMS were related to inclusion small quartz grain in sulfides. For image analysis, the surface \% of S and $\delta^{34} \mathrm{~S}$ form a better trend line but underestimate the error related to the volume of grain.

However, chalcopyrite form the residual ore zone $\left(\delta^{34} S:+1 \%\right)$ and pure chalcopyrite ovoid grains (XRD, $\delta^{34} \mathrm{~S}$ ca. $+11 \%$ ) from sandstone have different $\delta^{34} S$ values.

Similar to the residual sulfide-rich ore zone, large veins filled with dominant barite are present in the Uruguay open pit (CAM 9). The $\delta^{34} \mathrm{~S}$ of several barite veins varied from 12 to $17 \%$. V-CDT (Fig. $6 \mathrm{~A}$ ). Some centimeter-size barite crystals contain millimeter-size quartz, pyrite and chalcopyrite euhedral crystals (Fig. $5 \mathrm{H}$ ). Barite crystals from a large vein (CAM 9) have $\delta^{34} \mathrm{~S}$ values varying from 14.5 to $13.7 \%$ o and $\delta^{18} \mathrm{O}=12.7$ to $12.5 \%$. Euhedral quartz crystals in barite veins have $\delta^{18} \mathrm{O}$ values of 14.8 to $14.3 \%$.

Stable isotope results $\left(\delta^{13} \mathrm{C}\right.$ and $\left.\delta^{18} \mathrm{O}\right)$ in calcite and $\mathrm{Ca}-\mathrm{Mg}$ calcite vary from -1.7 to $-3 \%$ and 20 to $15 \%$, respectively (Table 3 ). The preliminary data show a $\delta^{13} \mathrm{C}$ difference between sandstone and conglomerate. Some of the stable isotope data have been obtained from cores previously examined in a fluid inclusion study (Ronchi et al., 2000).

\section{Discussion}

\subsection{Reconstruction of $\mathrm{Cu}-\mathrm{Fe} S$ chemical activity during sulfide and sulfate depositions}

Paragenetic reconstructions in the main ore zone (Laux et al. references therein) show successive sulfide, oxide and silicate precipitations. The chlorites, Fe, Cu-rich sulfides and barite hosting $\mathrm{Cl}$-rich hydrous fluid inclusions attest to large chemical changes that occurred throughout Uruguay ore history. Petrographic observation of sulfides (Py, Ссp, $\mathrm{Bn}$ ) and late barite indicate relative increases of $\mathrm{Cu}$ and $\mathrm{Ba}$ activities in veins or sedimentary units. The change from sulfide to barite indicates decreasing Fe and S activities controlled by an increasing oxygen activity and pH change (Chiba and Sakai, 1985; Gustafson, 1979; Gustafson and Hunt, 1975; Karadjov, 2003).

Experimental quantification of Ccp $+\mathrm{Py}+\mathrm{Bn}$ and Cc solubility in subcritical $\mathrm{NaCl}$ and $\mathrm{Na}-\mathrm{HS}+\mathrm{H}_{2} \mathrm{~S}$ fluids report that $\mathrm{Cu}$ is complexed as $\mathrm{Cu}(\mathrm{I})$ chloride in Na-rich solutions (Crerar and Barnes, 1976) and as dominant $\mathrm{Cu}(\mathrm{I})$-bisulfide in S-rich fluids (Archibald et al., 2002). The stability of $\mathrm{Cu}(\mathrm{I})$-bisulfide seems to increase with lowering temperature that may induce the precipitation of $\mathrm{Fe}$ and $\mathrm{Cu}$ as Py and $\mathrm{Ccp}$. Fe-Cu-rich sulfide precipitation might induce an increase in the $\mathrm{Cl}^{-}$and $\mathrm{H}^{+}$contents in fluid that would induce hydrolysis. Concomitantly, hydrolysis might be responsible for changes in the oxidation state (cations and anions) of the fluid (c.f. Chambefort et al., 2008; Hattori and Muehlenbachs, 1980; Richards, 2009; Rowins, 2000). In this hypothesis, the low thickness of the Camaquã sedimentary pile of immature sandstone combined with the magmatic origin of sulfur (Remus et al., 1999 , this study) suggest that the fluid contained sulfur as $\mathrm{H}_{2} \mathrm{~S}_{(\mathrm{aq})}-$ $\mathrm{HS}^{-}{ }_{\text {(aq) }}{ }^{-\mathrm{S}^{-}}{ }_{\text {(aq) }}$ rather than $\mathrm{SO}_{2}$ (Hattori and Muehlenbachs, 1980) and dissolved sulfate as $\mathrm{HSO}_{4}{ }^{-}$(aq) and/or $\mathrm{SO}_{4}{ }^{-}$(aq) depending on the $\mathrm{pH}$ and temperature.

Consequently, barite precipitation is related to either a high concentration of $\mathrm{Ba}^{++}$or $\mathrm{SO}_{4}^{-}$in solution, low $\mathrm{Ba} / \mathrm{SO}_{4}$ molar ratio in solution or $\mathrm{a} \mathrm{Ba} / \mathrm{SO}_{4}$ molar ratio higher than the saturation index produced at the oxic-anoxic boundary (e.g., Bolze et al., 1974; Breheret and Brumsack, 2000; Brumsack and Gieskes, 1983; Cecile et al., 1983; Dean and Schreiber, 1977; Hattori and Muehlenbachs, 1980). Barite saturation can occur at temperatures as low as $25{ }^{\circ} \mathrm{C}$ (Church and Wolgemuth, 1972; Edmond et al., 1979; Elderfield and Schultz, 1996; von Damm et al., 1985) to $250{ }^{\circ} \mathrm{C}$ (Glasby et al., 2006; Hanor, 2000; Lüders et al., 2001; Noguchi et al., 2011). Given the different processes of sulfide oxidation or sulfate reduction, we examined barite formation using $\mathrm{S}$ and O stable isotope values (e.g., Chiba and Sakai, 1985; Claypool et al., 1980; Kusakabe and Chiba, 1983; Kusakabe and Robinson, 1977; Ohmoto and Lasaga, 1982).

\subsection{Sulfur isotope source and temperature in large veins}

Sulfides in ore veins of the Uruguay mine produced pyrite and chalcopyrite values similar to those reported by Remus et al. (1999), which suggested a magmatic source of the sulfur (Watanabe and Sakai, 1983). However, $\delta^{34} \mathrm{~S}$ values of euhedral pyrite and euhedral chalcopyrite in veins suggest an unrealistic isotopic equilibrium at high temperatures $\left(>500{ }^{\circ} \mathrm{C}\right)$ that is not compatible with sediment metamorphic zonation or exsolution in sulfide grains. Moreover, barite veins including euhedral pyrite, chalcopyrite or ovoid polymetallic sulfides may precipitate from different $S$ isotopic sources. Petrographic observations of S-shape centimeter-size barite crystals indicate a ductile to brittle strain in crystals that may have produced oxygen isotopic exchange (c.f Hedenquist and Lowenstern, 1994; Simon and Ripley, 2011). This ductile deformation was also recorded through a secondary fluid assemblage entrapped at ca. $80{ }^{\circ} \mathrm{C}$. Consequently, we calculated the $\delta^{34} \mathrm{~S}_{\mathrm{SO}_{4}}$ (Kusakabe and Robinson, 1977) and $\delta^{18} \mathrm{O}_{\mathrm{H}_{2} \mathrm{O}}$ or $\delta^{18} \mathrm{O}_{\mathrm{SO}_{4}}$ values (Kusakabe and Robinson, 1977; Lloyd, 1968) of fluid in equilibrium with barite assuming a temperature of $80{ }^{\circ} \mathrm{C}$ ( secondary fluid inclusion; 


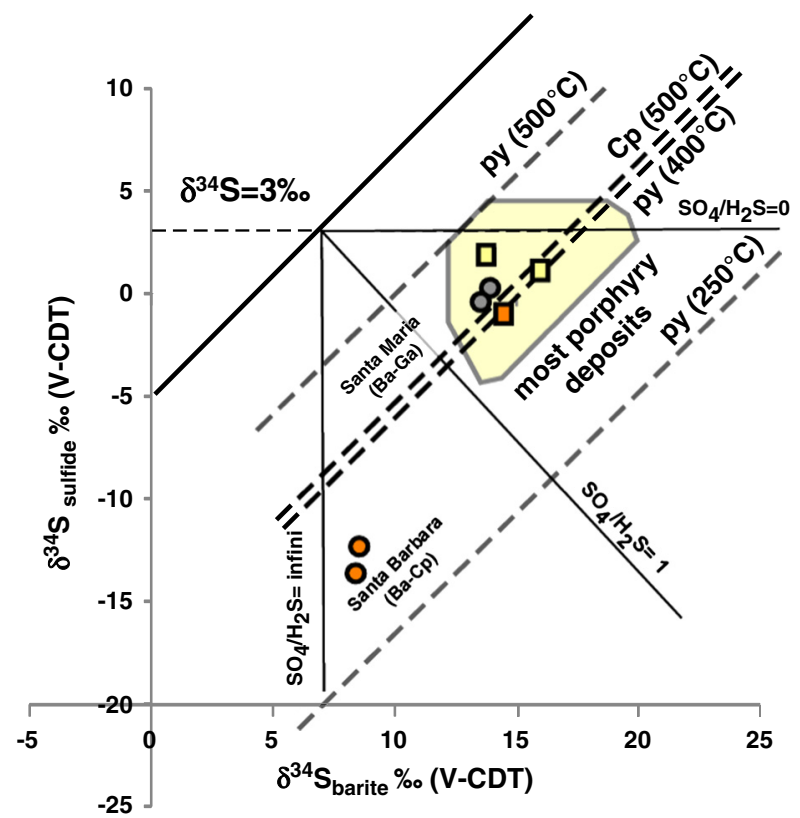

Fig. 7. Plot of $\delta^{34} S_{\text {barite }}$ vs. $\delta^{34} S_{\text {sulfides }}$ values from barite veins with euhedral pyrite and chalcopyrite centimeter-size crystals as well as selected acid-sulfate epithermal deposits (porphyry deposit fields from Simon and Ripley, 2011 and references therein; Santa-Maria and Barbara data from Remus et al., 2000). Also shown are the equilibrium fractionation lines at selected temperatures in the 500 to $250{ }^{\circ} \mathrm{C}$ range and the lines for $\delta^{34} \mathrm{~S}_{\Sigma \mathrm{S}}=0, \delta^{34} \mathrm{~S}_{\Sigma \mathrm{S}}=$ $+3 \%$ and variable $\mathrm{SO}_{4}^{2-} / \mathrm{H}_{2} \mathrm{~S}$ ratio of the parent fluids.

Fig. 9). The calculated $\delta^{34} \mathrm{~S}_{\mathrm{SO}_{4}}(-12 \%), \delta^{18} \mathrm{O}_{\mathrm{H}_{2} \mathrm{O}}(-12$ to $-4 \%$ ) and $\delta^{18} \mathrm{O}_{\mathrm{SO}_{4}}\left(8.6 \%\right.$ ) values of several $\mathrm{SO}_{4}$ complexes might produce barite when combined with stable isotope data derived from bacterial reduction $\left(\delta^{34} \mathrm{~S}_{\mathrm{SO}_{4}}\right)$, meteoric water $\left(\delta^{18} \mathrm{O}_{\mathrm{H}_{2} \mathrm{O}}\right)$ and/or seawater sulfate kinetic equilibrium $\left(\delta^{18} \mathrm{O}_{\mathrm{SO}_{4}}\right)$. Bacterial reduction and seawater sulfate may be compatible with a diagenetic system with oxic to anoxic reduction. However, the barite $\delta^{18} \mathrm{O} / \delta^{34} \mathrm{~S}$ ratio $(12.7 / 13.7$ or calculated $\left.\delta^{18} \mathrm{O}_{\mathrm{SO}_{4}} / \delta^{34} \mathrm{~S}_{\mathrm{SO}_{4}}=8.6 /-12\right)$ is not compatible with the modern ocean, bacterial sulfate reduction $\left(\delta^{18} \mathrm{O}_{\mathrm{SO}_{4}} / \delta^{34} \mathrm{~S}_{\mathrm{SO}_{4}}=9 / 21\right.$; c.f. Aharon and $\mathrm{Fu}$, 2000; Chiba and Sakai, 1985; Paytan et al., 2002) or diagenetic barite precipitation (Torres et al., 1996, 2002). Consequently, we considered hydrothermal barite and examined the magmatic source of sulfur at temperatures higher than $80{ }^{\circ} \mathrm{C} . \delta^{34} \mathrm{~S} / \delta^{18} \mathrm{O}$ stable isotope ratios that deviate from 4 suggest that oxygen isotopes of barite re-equilibrated with $\mathrm{H}_{2} \mathrm{O}$ at higher temperatures or were influenced by oxidized $\mathrm{H}_{2} \mathrm{~S}$. Therefore, we assumed that barite with euhedral pyrite and chalcopyrite precipitated from similar parent fluids. Consequently, the S stable isotope values of barite and sulfides remained unchanged by ductile deformation or $\mathrm{H}_{2} \mathrm{O}$ fluid circulation (secondary fluid inclusion). Under this assumption, the $\delta^{34} S_{\text {barite }}-\delta^{34} S_{\text {sulfides }}$ differences (Fig. 7; Rye, 2005) indicate temperatures between 500 and $400{ }^{\circ} \mathrm{C}$, a bulk sulfur isotope $\left(\delta^{34} \mathrm{~S}_{\Sigma \mathrm{S}}\right)$ value of $+3 \%$ and a $\mathrm{SO}_{4}{ }^{-}{ }_{(\mathrm{aq})} / \mathrm{H}_{2} \mathrm{~S}$ ratio between 0 and 1 . The $\delta^{34} \mathrm{~S}_{\Sigma S}$ of $+3 \%$ indicates $\mathrm{S}$ from a magmatic source but is higher than previously estimated values (Remus et al., 1999). The whole $\delta^{34} S_{\Sigma S}$ estimated from barite-sulfide ( $+3 \%$ ) or $\delta^{34} S_{\text {sulfide }}$ ( 1 to $4 \%$ ) are values reported for porphyry-type copper deposits (Ohmoto, 1986; Rye, 2005; Seal, 2006; Seal et al., 2000; Watanabe and Sakai, 1983). The large domain of $\delta^{34} S$ values ( 1 and $4 \%$ ) suggests several sulfur magmatic source or a single and isotopically evolved source of sulfur. However, temperatures between 500 and $400{ }^{\circ} \mathrm{C}$ do not support sulfide exsolution. Moreover, the calculated $\delta^{18} \mathrm{O}_{\mathrm{H}_{2} \mathrm{O}}$ value of barite at $400{ }^{\circ} \mathrm{C}$ is higher than the magmatic domain (Sheppard and Epstein, 1970; Taylor, 1968, 1987; Fig. 8). This unrealistic calculated $\delta^{18} \mathrm{O}_{\mathrm{H}_{2} \mathrm{O}}$ value combined with the ductile aspect of barite crystals suggest oxygen isotope exchanges associated with deformation that may be related to the entrapment of aqueous fluid inclusions (Ronchi et al., 2000).

Phase diagrams from the literature ( 350 to $250{ }^{\circ} \mathrm{C}$ : Heald et al., 1987; Ohmoto, 1986; Ohmoto and Rye, 1979) indicate that pyrite, chalcopyrite and barite assemblages can occur for sulfur at $10^{-8.5} \mathrm{~atm}$ and oxygen fugacities between $10^{-32}$ to $10^{-35}$ atm. These $\mathrm{O}$ and $\mathrm{S}$ activity/fugacity values would allow quartz formation under low acidic to neutral pHs $\left(4.3<\mathrm{H}_{2} \mathrm{~S}_{\mathrm{aq}}<7.8\right.$ ), which is compatible with the $\mathrm{SO}_{4} / \mathrm{H}_{2} \mathrm{~S}$ ratio between 0 and 1 determined from the interpretation of baritesulfide $\mathrm{S}$ isotopes.

\subsection{Sulfur source and thermometry in sandstone}

Stable isotope data from sulfide in sandstone differ from those of large veins. Moreover, petrographic observation in sandstone suggests a sequence of precipitation and replacement with Py, broken Py replaced by Ccp and Ccp with rims of Bn. Consequently, Py, Ccp and Bn precipitations may have been produced from different sources of sulfur, copper and iron, unrelated to grain size or depth. Therefore, $\delta^{34} \mathrm{~S}$ values form a single mixing line from pure Py to pure Ccp grains. This binary mixing line indicates one episode of Py and Ccp precipitation rather

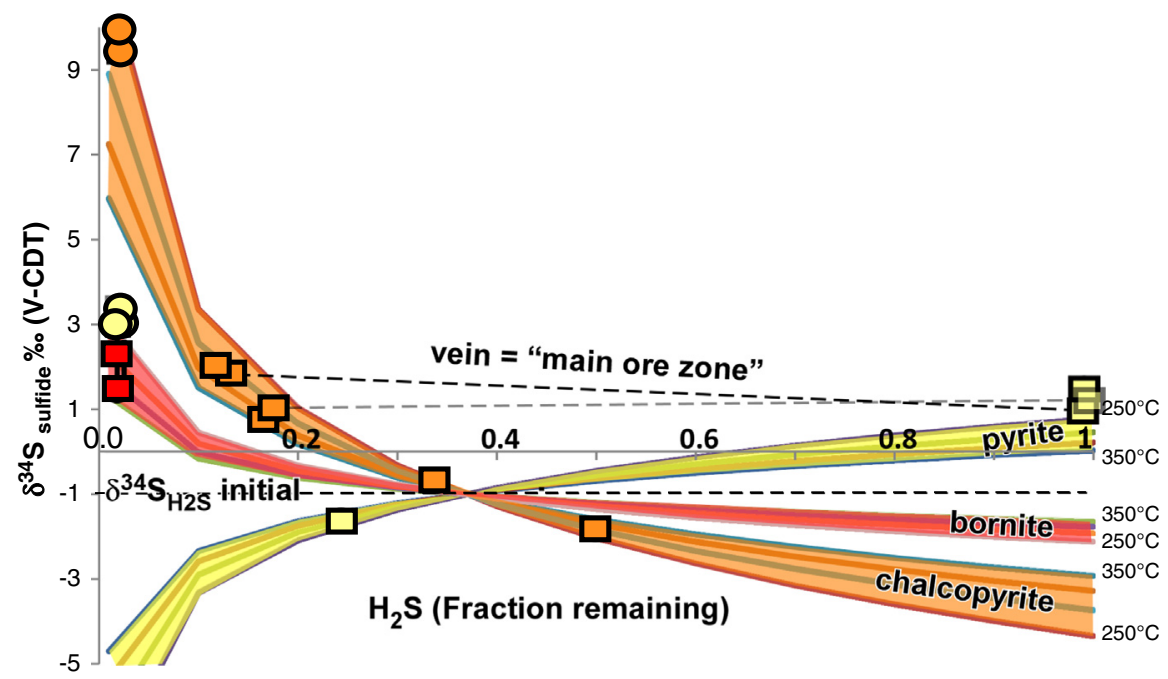

ore in veins

ore in sandstone

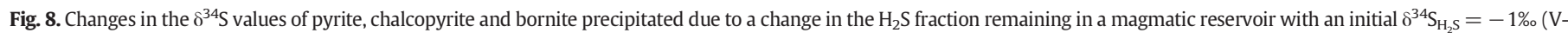

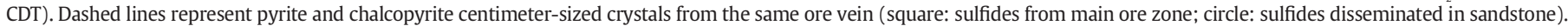


than several episodes. We consider a crystallization sequence of pyrite, followed by chalcopyrite and then bornite and examine a fractional crystallization (FC) model associated with different sulfur magmatic reservoirs $\left(\delta^{34} \mathrm{~S}\right)$. This fractional crystallization model assumes that the first S flux (impulse) was FeS-rich and came from a $\mathrm{S}$ magmatic reservoir. Later S-rich fluids were $\mathrm{Cu}>\mathrm{Au}$ - and $\mathrm{Pb}$-enriched with increasing oxygen fugacity and/or $\mathrm{pH}$. Therefore, $\delta^{34} \mathrm{~S}$ values in pyrite, chalcopyrite and bornite were calculated for temperatures varying from 350 to $250{ }^{\circ} \mathrm{C}$, an initial $\delta^{34} \mathrm{~S}_{\mathrm{H}_{2} \mathrm{~S}}$ of $-1 \%$ o close to the magmatic source and an $\mathrm{S}$ fractionation factor of $\mathrm{H}_{2} \mathrm{~S}$-pyrite, $\mathrm{H}_{2} \mathrm{~S}$-chalcopyrite or $\mathrm{H}_{2} \mathrm{~S}$-bornite (Li and Liu, 2006; Fig. 8). In this FC model, $\Delta^{34}$ S pyrite-chalcopyrite from joined grains in an ore vein indicates that pyrite then chalcopyrite formation could have precipitated from a single $S$ isotope reservoir $\left(\delta^{34} \mathrm{~S}_{\mathrm{H}_{2} \mathrm{~S}}:-1\right)$, and the initial S reservoir would have lost 90 to $50 \%$ of its initial $\mathrm{H}_{2} \mathrm{~S}$ to precipitate chalcopyrites ( 0.1 to 0.5 of $\mathrm{H}_{2} \mathrm{~S}$ remaining fraction). In this model, chalcopyrite would form after a large pyrite deposition. This hypothesis is supported by mining observations (Ronchi et al., 2000) that found a 60/40 pyrite/chalcopyrite proportion in Uruguay mine ore.

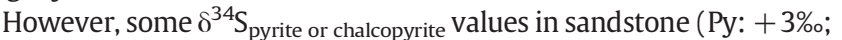
Ccp: $10.5 \%$ ) or Santa Barbara ore (e.g., Remus et al., 2000; Ccp: -13 to $-15 \%$ and Ba: 8.5 to $7.5 \%$ ) could not have formed from $\mathrm{H}_{2} \mathrm{~S}$-rich fluid with a unique $\delta^{34} \mathrm{~S}$ value of $-1 \%$ or lower temperature of formation. Consequently, we explored a pyrite-chalcopyrite precipitation for temperatures lower than $<250{ }^{\circ} \mathrm{C}$ and an initial $\delta^{34} \mathrm{~S}_{\mathrm{H}_{2} \mathrm{~S}}$ of $-1 \%$. The FC model never predicted measured $\delta^{34} \mathrm{~S}$ values (negative), which demonstrates a different $\delta^{34} \mathrm{~S}_{\mathrm{H}_{2} \mathrm{~S}}$ reservoir for sediments $(\neq-1 \%)$ than the main ore zone. We also examined the FC model for ovoid sulfides precipitated in sandstone. $\Delta^{34} \mathrm{~S}$ pyrite-chalcopyrite differences indicate either a high $\mathrm{H}_{2} \mathrm{~S}$ loss precipitated as pyrite $\left(<0.1\right.$ of $_{2} \mathrm{~S}$ remaining fraction; Fig. 8) or highly fractionated $\mathrm{H}_{2} \mathrm{~S}$ fluids in equilibrium with chalcopyrite or temperature lower than $250{ }^{\circ} \mathrm{C}$. These hypotheses
( $\mathrm{H}_{2} \mathrm{~S}$ loss or isotope-fractionated sulfur and temperature) would then be associated with porosity or impermeability in sandstones compared to fractures (main ore zone; $\delta^{34} \mathrm{~S}_{\mathrm{H}_{2} \mathrm{~S}}$ : $-1 \%$ ). However, the minimal temperature would be bracketed by examination of chlorite on quartz coating some sulfide or chlorite veins that mark late fluid circulation.

\subsection{Silicates and late hydrous fluids}

Among the alteration phases, chlorite is the most ubiquitous silicate. Fe-clinochlore, $\mathrm{Mg}$-Fe chamosite and $\mathrm{Mg}$-chamosite as well as quartz and hematite indicate a high $\mathrm{Mg}-\mathrm{Fe}-\mathrm{Si}$ activity associated with $\mathrm{H}_{2} \mathrm{O}$ rich fluids compared to sulfide-sulfate precipitation $\left(\mathrm{H}_{2} \mathrm{~S}-\mathrm{SO}_{4}-\mathrm{H}_{2} \mathrm{O}\right)$. These chlorites with different polytypes could form from a low to high temperature hydrothermal system (Inoue et al., 2010; Zang and Fyfe, 1995). Chamosite and clinochlore in pelitic units suggest Mgenrichment and $\mathrm{Fe} / \mathrm{Fe}+\mathrm{Mg}$ change related to hydrothermal fluid (Laux et al., 2005). Moreover, chlorite polytypes indicate layer ordering at high temperatures ( $>150{ }^{\circ} \mathrm{C}$ : Bailey, 1988; Walker, 1993). However, the temperature calculation of chlorite depends on the whole rock composition, pressure and temperature (Vidal et al., 2001; Walshe, 1986) and $\mathrm{Al}^{\mathrm{VI}}-\mathrm{Fe}^{3+}$ contents (Cathelineau, 1988; Inoue et al., 2010). We choose the geothermometer developed by Inoue et al. (2010) applied to sedimentary rocks, active geothermal fields, low-grade metamorphism and Fe-rich chlorite.

Differences between geothermometers are related to the cation distribution in the structural formula of chlorite. Temperature ranges were calculated with average electron microprobe data and a $\mathrm{Fe}^{3+} / \mathrm{Fe}_{\text {tot }}$ ratio from 0.1 to 0.2 (Table 1 ).

They vary from ca. 140 (PT-45-14, Mg-clinochlore) to $310{ }^{\circ} \mathrm{C}$ (CAM 1, CAM12). The present estimate of temperature range is wider than the chlorite temperature reported by Lima (1998) using the thermometer proposed by Cathelineau (1988). Temperature changes are related

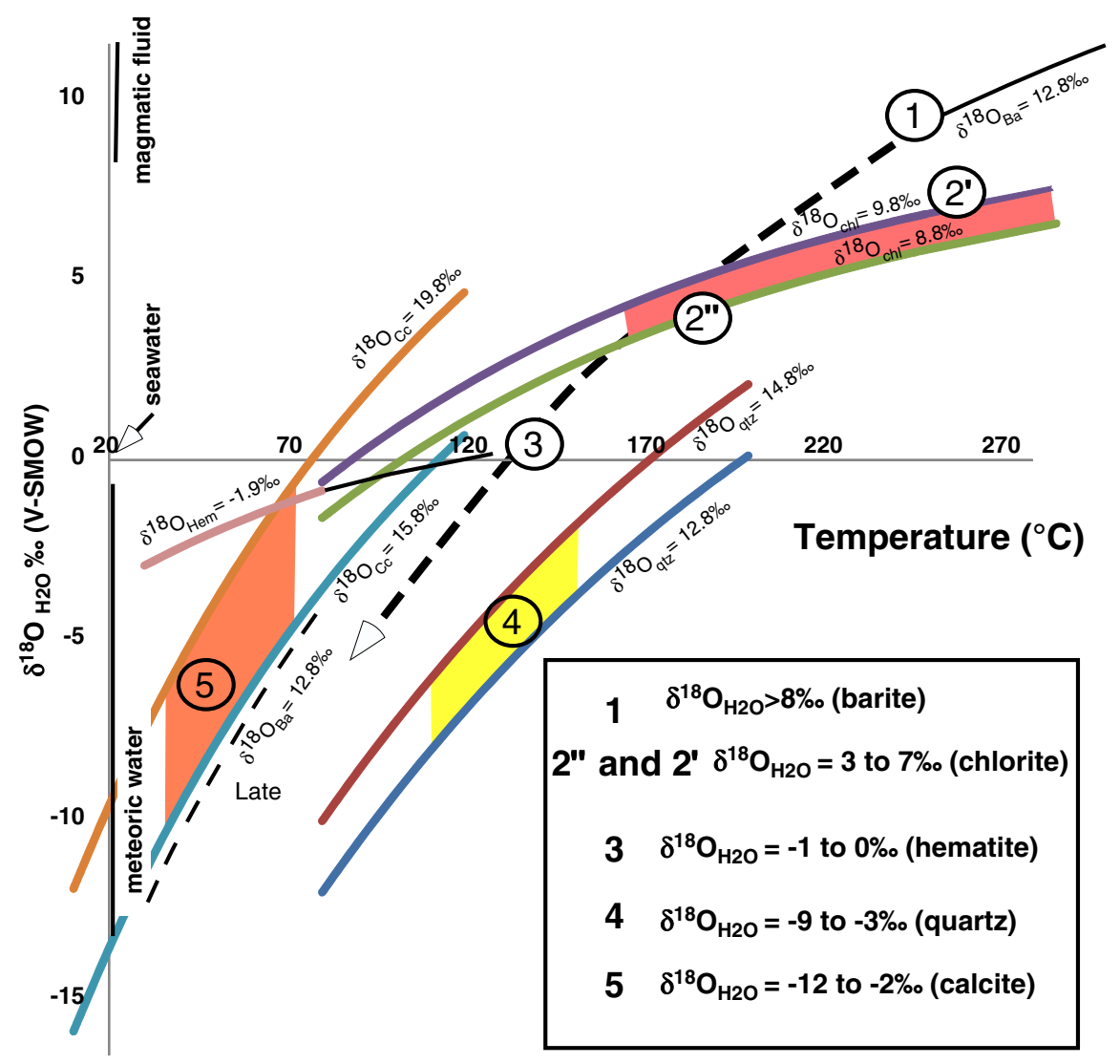

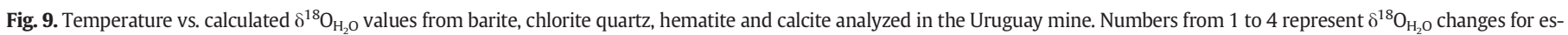
timated (barite), calculated (chlorite) or measured temperatures (fluid inclusions of quartz and calcite). 
to thermometers (Cathelineau and Inoue). A temperature of $310{ }^{\circ} \mathrm{C}$ calculated (Lima, 1998) using the method suggested by Cathelineau (1988) is equivalent to temperatures of $260\left(\mathrm{Fe}^{3+} / \mathrm{Fe}_{\text {tot }}\right.$ ratio of 0.2$)$ and $290{ }^{\circ} \mathrm{C}\left(\mathrm{Fe}^{3+} / \mathrm{Fe}_{\text {tot }}\right.$ ratio of 0.1 ; Table 1$)$ using the method presented by Inoue et al. (2010). There are two extreme cases; a) $\mathrm{Fe}^{3+}=\mathrm{Fe}^{2+}$, which does not agree with the XRD pattern $\left(\mathrm{Fe}^{2+}>\mathrm{Fe}^{3+}\right)$ and b) all iron as $\mathrm{Fe}^{2+}$, which will lead to a too large a number of vacant sites for a chlorite structure. Temperatures would have to have been 30 to $50{ }^{\circ} \mathrm{C}$ lower (a) or 50 to $100{ }^{\circ} \mathrm{C}$ higher (b). Regardless of large errors of temperature, a reasonable $\mathrm{Fe}^{3+} / \mathrm{Fe}_{\text {tot }}$ content indicating temperatures varying from 310 to $145{ }^{\circ} \mathrm{C}$ for chlorite does not seem to be related to a specific chemical composition (Table 1 ) or chlorite polytype (Ib or IIb). Nevertheless, the PS45-1-14 m chlorite vein composed of $\mathrm{Mg}-\mathrm{Fe}$ chamosite (IIb polytype) has the lowest estimated temperature (ca. $145^{\circ} \mathrm{C}$ ).

Therefore, we used 310 to $145{ }^{\circ} \mathrm{C}$ (Table 1 ) with $\mathrm{O}$ and $\mathrm{H}$ stable isotopes data (PSU45-1-14 m; PT-49 and CAM 12) to calculate the $\delta^{18} \mathrm{O}_{\mathrm{H}_{2} \mathrm{O}}$ and $\delta \mathrm{D}_{\mathrm{H}_{2} \mathrm{O}}$ in equilibrium chlorite (oxygen fractionation factor: Savin and Lee, 1988; hydrogen isotopes fractionation factor: Capuano, $1992)$. The calculated $\delta{ }^{18} \mathrm{O}_{\mathrm{H}_{2} \mathrm{O}}$ and $\delta \mathrm{D}_{\mathrm{H}_{2} \mathrm{O}}$ vary from +7 to $+3 \%$ 。 (Fig. 9) and -35 to $-45 \%$, respectively. Regardless of temperature uncertainty, calculated $\delta^{18} \mathrm{O}$ and $\delta \mathrm{D}$ values of $\mathrm{H}_{2} \mathrm{O}$ are located between the magmatic and metamorphic domain described by Sheppard and Epstein (1970) and Taylor (1968). This stables isotopes values far from the meteoric water line suggest a meteoric water/magmatic rock interaction or a small proportion of meteoric water mixed with a magmatic fluid. For the specular hematite crystals collected with chlorite (Fe-chamosite CAM 12; CAM 11), no thermometer or paragenetic sequence is available. We assumed that hematite could have formed from 310 to $80{ }^{\circ} \mathrm{C}$. Moreover, the hematite- $\mathrm{H}_{2} \mathrm{O}$ oxygen fractionation factor (Bao and Koch, 1999) is not well constrained for a temperature lower than $150{ }^{\circ} \mathrm{C}$. Nevertheless, for a temperature domain between 150 and $310{ }^{\circ} \mathrm{C}$, the calculated $\delta^{18} \mathrm{O}_{\mathrm{H}_{2} \mathrm{O}}$ in equilibrium with hematite would vary from +4 to $-2 \%$. Similarly, chlorite vein and sandstone cement are associated with disseminated quartz overgrowth and or euhedral quartz in veins. Quartz crystallization temperatures between 150 and $110{ }^{\circ} \mathrm{C}$ and $<80^{\circ} \mathrm{C}$ for calcite measured by fluid inclusion microthermometry (Table 3 ) were used to calculate $\delta^{18} \mathrm{O}_{\mathrm{H}_{2} \mathrm{O}}$ in equilibrium. Calculated $\delta^{18} \mathrm{O}_{\mathrm{H}_{2} \mathrm{O}}$ varies from -3 to $-9 \%$ for quartz and -2 to $-12 \%$ for calcite (oxygen fractionation factor $=$ quartz $-\mathrm{H}_{2} \mathrm{O}$ : Clayton et al., 1972; calcite- $\mathrm{H}_{2} \mathrm{O}$ : Kim and O'Neil, 1997; Fig. 9). Moreover,

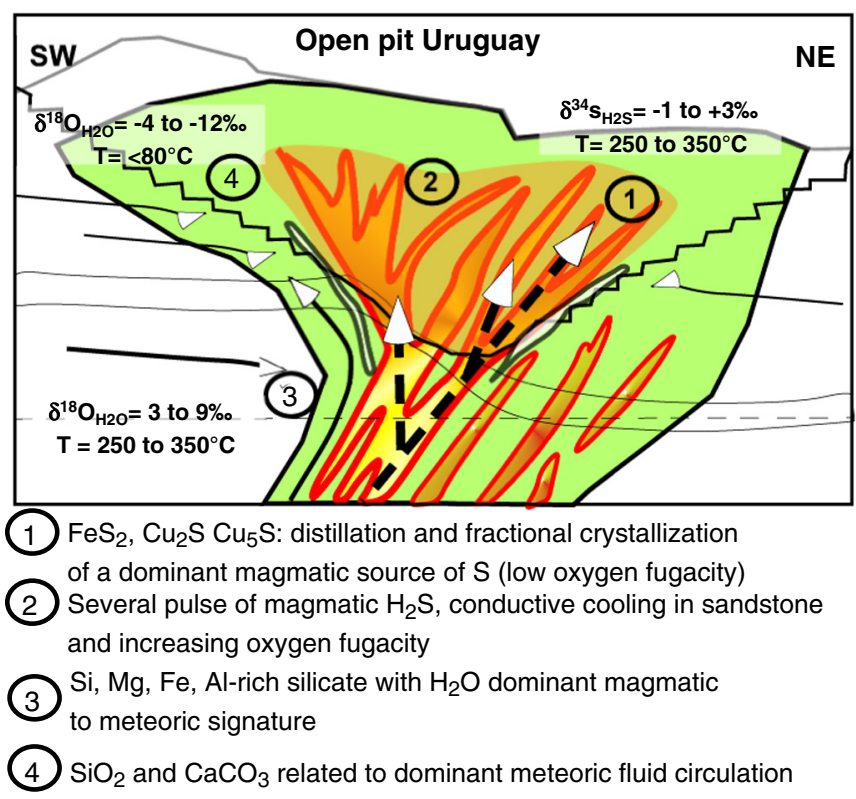

Fig. 10. Synthetic cross-section of the Uruguay open pit with temperatures, $\delta^{34} \mathrm{~S}_{\mathrm{H}_{2}} \mathrm{~S}$ and $\delta^{18} \mathrm{O}_{\mathrm{H}_{2} \mathrm{O}}$ of fluids involved the $\mathrm{Cu}-\mathrm{Au}$ ore deposit. different $\delta^{13} \mathrm{C}_{\mathrm{CaCO}_{3}}$ values for calcite cements in sandstone and conglomerate units indicate two slightly different sources of inorganic carbon, suggesting different stages of calcite precipitation associated with meteoric recharge in the area (e.g., Bongiolo et al., 2011) and of the Camaquã Basin.

\section{Conclusions}

From the sequence of sulfides, chlorite, barite and calcite we propose a schematic of the chemical origin of fluids and temperature evolution of the Uruguay mine (Fig. 10). The reconstruction of the sulfide and sulfate paragenetic sequence of pyrite, chalcopyrite and bornite associated with different values of $\delta^{34} \mathrm{~S}$ suggest that the ore precipitated from several pulses of mineralizing fluids with sulfur of magmatic origin. The different pulses were associated with $\mathrm{Cu}$ enrichment and bornite precipitation in sandstone and veins. Moreover, the pervasive occurrence of $\mathrm{Fe}-\mathrm{Mg}$ chlorites coating grains of sandstone and conglomerate or barite and calcite veins indicate porosity reduction in sandstone and fractures with fluid circulation associated with $\mathrm{H}_{2} \mathrm{O}-\mathrm{HS}-\mathrm{SO}_{4} /$ rock interaction in slightly acidic conditions. The Fe-Mg chlorite, barite and calcite precipitations were related to decreasing temperatures varying from 310 to $80{ }^{\circ} \mathrm{C}$ and calculated $\delta{ }^{18} \mathrm{O}_{\mathrm{H}_{2} \mathrm{O}}$ and $\delta \mathrm{D}_{\mathrm{H}_{2} \mathrm{O}}$ values that cover a range from a magmatic fluid ( $+9 \%$; barite and chlorite) to meteoric hydrothermal fluids ( -3 to $-12 \%$; quartz and calcite). We interpreted temperature and chemical isotope changes as a $\mathrm{H}_{2} \mathrm{O}-\mathrm{HS}_{-} \mathrm{SO}_{4} /$ rock interaction combined cooling magmatic $\mathrm{H}_{2} \mathrm{O}$ and S-enriched fluids with S-poor meteoric fluids infiltrated throughout the sediment (Fig. 10).

\section{Acknowledgments}

This study was partly funded by "Conselho Nacional de Desenvolvimento Cientifico e Tecnologico - CNPq", CAPES - COFECUB project (619-08/2008) and a personal grant to C. Renac from Université de Saint-Etienne. The authors thank CBC, CPRM and the VOTORANTIN metal company for borehole samples. We thank Thomas Ulrich and Roberto Perez Xavier for reviews of earlier versions of the manuscript and constructive comments.

\section{References}

Aharon, P., Fu, B., 2000. Microbial sulfate reduction rates and sulfur and oxygen isotope fractionations at oil and gas seeps in deep water Gulf of Mexico. Geochim. Cosmochim. Acta 64 (2), 233-246.

Almeida, D.P.M., Zerfass, H., Basei, M.A., Petry, K., Gomes, C.H., 2002. The Acampamento Velho Formation, a Lower Cambrian bimodal volcanic package: geochemical and stratigraphic studies from the Cerro do Bugio, Perau and Serra de Santa Bárbara (Caçapava do Sul, Rio Grande do Sul, RS - Brazil). Gondwana Res. 5, 721-733.

Almeida, D.P.M., Zerfass, H., Basei, M.A., Lopes, R.C., 2003. Eventos vulcânicos alcalinos na Bacia do Camaquã: o vulcanismo Neoproterozoico III Acampamento Velho e o magmatismo Meso-Ordoviciano (?) Rodeio Velho. In: Ronchi, L.H., Althoff, F. (Eds.), Caracterização e Modelamento de Depósitos Minerais. Universidade do Vale do Rio dos Sinos, São Leopoldo, pp. 325-350.

Almeida, D.P.M., Conceicão, R.V., Chemale Jr., F., Koester, E., Borba, A., Petry, K., 2005 Evolution of heterogeneous mantle in the Acampamento Velho and Rodeio Velho volcanic events, Camaquã Basin, southern Brazil. Gondwana Res. 8, 479-492.

Archibald, S.M., Migdisov, A.A., Williams-Jones, A.E., 2002. An experimental study of the stability of copper chloride complexes in water vapor at elevated temperatures and pressures. Geochim. Cosmochim. Acta 66 (9), 1611-1619.

Bailey, S.W., 1980. Summary of recommendation of AIPEA Nomenclature Committee. Clay Clay Miner. 15, 85-93.

Bailey, S.W., 1988. Chlorites: structure and crystal chemistry. Mineralogical Society of America Reviews in Mineralogy, 19, pp. 347-403.

Bailey, S.W., Brown, B.E., 1962. Chlorite polytypism: I. Regular and semi-random onelayer structures. Am. Mineral. 47, 819-850.

Bao, H., Koch, P.L., 1999. Oxygen isotope fractionation in ferric oxide-water systems: low temperature synthesis. Geochim. Cosmochim. Acta 63, 599-613.

Basei, M.A.S., Siga Jr., O., Masquelin, H., Harara, O.M., Reis Neto, J.M., Preciozzi, F.P., 2000 The Dom Feliciano Belt of Brasil and Uruguay and its foreland domain, the Rio de La Plata Craton: framework, tectonic evolution and correlation with similar provinces of southwestern Africa. In: Cordani, U.G., Milani, E.J., Thomaz Filho, A., Campos, D.A (Eds.), Tectonic Evolution of South America. 31st International Geological Congress, Rio de Janeiro, pp. 311-334.

Bayliss, P., 1975. Nomenclature of the trioctahedral chlorite. Can. Mineral. 13, 178-180. 
Bettencourt, J.S., 1972. A Mina de cobre de Camaquã. (Rio Grande do Sul. Tese de Doutorado) Universidade de São Paulo, São Paulo (175 pp.)

Bigeleisen, J., Perlman, M.L., Prosser, H.C., 1952. Conversion of hydrogenic materials to hydrogen for isotopic analysis. Anal. Chem. 24, 1356-1357.

Bitencourt, M. de F., Nardi, L.S.V., 2000. Tectonic setting and sources of magmatism related to the Southern Brazilian Shear Belt. Rev. Brasil. Geocienc. 30 (1), 186-189.

Bolze, C.E., Malone, P.G., Smith, M.J., 1974. Microbial mobilization of barite. Chem. Geol. 13, 141-143.

Bongiolo, M.E., Renac, C., Mexias Sampaio, A., Gomes Boscato, M.E., Ronchi, L., Patrier-Mas, P., 2011. Evidence of Ediacaran glaciation in southernmost Brazil through magmatic to meteoric fluid circulation in the porphyry-epithermal $\mathrm{Au}-\mathrm{Cu}$ deposits of Lavras do Sul. Precambrian Res. 189 (3-4), 404-419.

Borba, A.W., 2006. Evolução geológica da "Bacia do Camaquã" (Neopropterozóico e Paleozóico inferior do Escudo Sul-rio-grandense, RS, Brasil): uma visão com base na integração de ferramentas de estratigrafia, petrografia e geologia isotópica. (Tese de Dotourado) Universidade Federal do Rio Grande do Sul (110 pp.)

Borba, A.W., Maraschin, A.J., Mizusaki, A.M.P., 2007. Evolução tectono-estratigráfica e paleoclimática da Formação Maricá (Escudo Sul-rio-grandense, Brasil): um exercício de geologia histórica e análise integrada de uma bacia sedimentar neoproterozóica. Pesqui. Geociênc. (UFRGS) 34, 57-74.

Breheret, J.-G., Brumsack, H.J., 2000. Barite concretions as evidence of pauses in sedimentation in the Marnes Bleues Formation of Vocontian Basin (SE France). Sediment. Geol. 130, 205-228.

Brito Neves, B.B., Cordani, U.G., 1991. Tectonic evolution of South America during the Late Proterozóic. Precambrian Res. 53, 34-40.

Brumsack, H.J., Gieskes, J.M., 1983. Interstitial water trace-metal chemistry of laminated sediments from the Gulf of California, Mexico. Mar. Chem. 14, 89-106.

Capuano, R.M., 1992. The temperature dependence of hydrogen isotope fractionation between clay minerals and water: evidence from a geopressured system. Geochim. Cosmochim. Acta 56, 2547-2554

Cathelineau, M., 1988. Cation site occupancy in chlorites and illites as a function of temperature. Clay Miner. 23, 471-485.

Cecile, M.P., Shakur, M.A., Krouse, H.R., 1983. The isotopic composition of Western Canadian barites and the possible derivation of oceanic sulfate $\delta^{34} \mathrm{~S}$ and $\delta^{18} \mathrm{O}$ age curves. Can. J. Earth Sci. 20, 1528-1535.

Chambefort, I., Dilles, J.H., Kent, A.J.R., 2008. Anhydrite-bearing andesite and dacite as a source for sulfur in magmatic-hydrothermal mineral deposits. Geology 36, 719-722.

Chemale Jr., F., 2000. Evolução Geológica do Escudo Sul-Riograndense. In: Holz, M., De Ros, L.F. (Eds.), Geologia do Rio Grande do Sul, Porto Alegre, RS, pp. 13-52.

Chemale Jr., F., 1997. Tectonic Evolution of Southern Brazil and Southern Africa during the Neoproterozoic and Early Paleozoic. 14th International Conference on Basement Tectonics, Ouro Preto, pp. 10-11.

Chiba, H., Sakai, H., 1985. Oxygen isotope exchange between dissolved sulfate and water at hydrothermal temperatures. Geochim. Cosmochim. Acta 49, 993-1000.

Church, T.M., Wolgemuth, K., 1972. Marine barite saturation. Earth Planet. Sci. Lett. 15 35-44.

Claypool, G.E., Holser, W.T., Kaplan, I.R., Sakai, H., Zak, I., 1980. The age curves of sulfur and oxygen isotopes in marine sulfate and their mutual interpretation. Chem. Geol. 28, 199-260.

Clayton, R.N., Mayeda, T.K., 1963. The use of bromine pentafluoride in the extraction of oxygen from oxides and silicates for isotopic analysis. Geochim. Cosmochim. Acta $27,43-52$.

Clayton, R.N., O'Neil, J.R., Mayeda, T.K., 1972. Oxygen isotope exchange between quartz and water. J. Geophys. Res. 77, 3057-3067.

Coplen, T.B., 1988. Normalization of oxygen and hydrogen isotope data. Chem. Geol. Isot. Geosci. Sect. 72, 293-297.

Craig, H., 1953. The geochemistry of the stable carbon isotopes. Geochim. Cosmochim. Acta 3, 53-92.

Crerar, D., Barnes, H., 1976. Ore solution chemistry V. Solubilities of chalcopyrite and chalcocite assemblages in hydrothermal solutions at $200{ }^{\circ} \mathrm{C}$ to $350{ }^{\circ} \mathrm{C}$. Econ. Geol. 71 , 772-794.

da Silva, L.C., Hartmann, L.A., McNaughton, N.J., Fletcher, I., 1999. Zircon U-Pb SHRIMP dating of a Neoproterozoic overprint in Paleoproterozoic granitic-gneissic terranes, southern Brazil. Am. Mineral. 85 (5-6), 649-667.

Dean, W.E., Schreiber, B.C., 1977. Authigenic barite. In: Lancelot, Ed Y., et al. (Eds.), Proceedings of the Deep Sea Drilling Project. Initial reports, 41. U.S. Government Printing Office, Washington, D.C., pp. 915-931.

Edmond, J.M., Measures, C., McDuff, R.E., Chan, L.H., Collier, R., Grant, B., Gordon, L.I. Corliss, J.B., 1979. Ridge crest hydrothermal activity and the balances of the major and minor elements in the ocean: the Galapagos data. Earth Planet. Sci. Lett. 46, 1-18

Elderfield, H., Schultz, A., 1996. Mid-ocean ridge hydrothermal fluxes and the chemical composition of the ocean. Annu. Rev. Earth Planet. Sci. 24, 191-224.

Faccini, U.F., Paim, P.S.G., Fragoso-Cesar, A.R.S., 1987. Análise faciológica das molassas brasilianas na região das minas do Camaquã, RS. Simp. Sul-Bras. Geol., 3. Curitiba, Atas, vol. 1. SBG, pp. 75-91.

Foster, M.D., 1962. Interpretation of the composition and a classification of the chlorites. U. S. Geol. Surv. Prof. Pap. 414-A, 1-33.

Fragoso-Cesar, A.R.S., 1980. O Cráton do Rio de La Plata e cinturão Don Feliciano no Escudo Uruguaio-sul-riograndense. Cong. Brás. Geol., 31. Balneário de Camburiú, Anais, vol. 5. SBG, pp. 2879-2892

Fragoso-César, A.R.S., Machado, R., Sayeg, H.S., Fambrini, G.L., 1992. Bacias orogênicas do Ciclo Brasiliano no Rio Grande do Sul e Uruguai. Workshop sobre as bacias molássicas brasilianas. Boletim de Resumos Expandidos, pp. 47-53.

Glasby, G.P., Cherkashov, G.A., Gavrilenko, G.M., Rashidov, V.A., Slovtsov, I.B., 2006. Submarine hydrothermal activity and mineralization on the Kurile and western Aleutian Island arcs, NW Pacific. Mar. Geol. 231, 163-180.
Gonzalez, M., Teixeira, G., 1980. Considerações sobre estratigrafia e ambientes de sedimentação da Região das Minas do Camaquã e Jazida Santa Maria-RS. Congresso Brasileiro de Geologia 31. Anais, vol. 3. Sociedade Brasileira de Geologia, SBG, Balneário de Camboriu, pp. 1513-1524.

Gross, A.O.M.S., Porcher, C.C., Fernandes, L.A.D., Koester, E., 2006. Neoproterozoic low-pressure/high temperature collisional metamorphic evolution in the Varzea do Capivarita Metamorphic Suite, SE Brazil: thermobarometric and Sm/Nd evidence. Precambrian Res. 147, 41-64.

Gustafson, L.B., 1979. Porphyry copper deposits and calc-alkaline volcanism. In: Mc Elhinny, M.W. (Ed.), The Earth: Its Origin, Structure and Evolution. Academic Press, London, pp. 427-468.

Gustafson, L.B., Hunt, J.P., 1975. The porphyry copper deposit at El Salvador, Chile. Econ. Geol. 70, 857-912.

Hanor, J.S., 2000. In: Alpers, C.N., Jambor, J.L., Nordstrom, D.K. (Eds.), Barite-celestine geochemistry and environments of formation. Reviews in Mineralogy \& Geochemistry Sulfate Minerals, 40. Mineralogical Society of America, Washington, D.C., pp. $193-275$

Hartmann, L.A., 1998. Deepest exposed crust of Brazil: geochemistry of Paleoproterozoic depleted Santa Maria Chico granulites. Gondwana Res. 1 (3/4), 331-341.

Hartmann, L.A., Chemale Jr., F., Philipp, R.P., 2007. Evolução Geotectônica do Rio Grande do Sul no Pré-Cambriano. In: Iannuzzi, R., Frantz, J.C. (Eds.), Geologia do Rio Grande do Sul. Porto Alegre, pp. 99-123.

Hattori, K., Muehlenbachs, K., 1980. Marine hydrothermal alteration at a Kuroko ore deposit, Kosaka, Japan. Contr. Mineral. Petrol. 74, 285-292.

Hayes, J.B., 1970. Polytypism of chlorite in sedimentary rocks. Clay Clay Miner. 18, 285-306.

Heald, P., Foley, N., Hayba, D., 1987. Comparative anatomy of volcanic-hosted epithermal deposits: acid-sulfate and adularia-sericite types. Econ. Geol. 82 (1), 1-26.

Hedenquist, J.W., Lowenstern, J.B., 1994. The role of magmas in the formation of hydrothermal ore deposits. Nature 370, 519-527.

Inoue, A., Kurokawa, K., Tamao Hatta, T., 2010. Application of chlorite geothermometry to hydrothermal alteration in Toyoha geothermal system, southwestern Hokkaido, Japan. Resour. Geol. 60 (1), 52-70.

Janikian, L., 2004. Sequuências deposicionais e evolução paleoambiental do Grupo Bom Jardim e da Formação Acampamento Velho, Supergrupo Camaquã, Rio Grande do Sul. Unpublished Doctoral Thesis. IGc-USP, São Paulo, 189 pp.

Janikian, L., Almeida, R.P., Fragoso-Cesar, A.R.S., Fambrini, G.L., 2003. Redefinição do Grupo Bom Jardim (Neoproterozóico III) em sua área-tipo: litoestratigrafia, evolução paleoambiental e contexto tectônico. Rev. Brasil. Geocienc. 33, 349-362.

Janikian, L., Almeida, R.P., Fragoso-Cesar, A.P.M.R., 2005. Evolução paleoambiental e sequuências deposicionais do Grupo Bom Jardim e Formação Acampamento Velho (Supergrupo Camaquã) na porção norte da Sub-Bacia Camaquã Ocidental. Rev. Brasil. Geocienc. 35, 245-256.

Janikian, L., Paes de Almeida, R., Ferreira da Trindade, R.I., Fragoso-Cesar, A.R.S., D'AgrellaFilho, M.S., Dantas, E.L., Tohver, E., 2008. The continental record of Ediacaran volcanosedimentary successions in southern Brazil and their global implications. Terra Nova 20 (4), 259-266.

Karadjov, M., 2003. Phase diagram of the Fe-Cu-S-O system at $\mathrm{T}=400{ }^{\circ} \mathrm{C}$ and $\mathrm{P}=$ 1 kbar. C. R. Acad. Bulg. Sci. 56 (8), 43.

Kim, S.T., O'Neil, J.R., 1997. Equilibrium and nonequilibrium oxygen isotope effects in synthetic carbonates. Geochim. Cosmochim. Acta 61, 3461-3475.

Kusakabe, M., Chiba, H., 1983. Oxygen and sulfur isotope composition of barite and anhydrite from the Fukazawa deposit, Japan. Econ. Geol. Monogr. 5, 292-301.

Kusakabe, M., Robinson, B.W., 1977. Oxygen and sulfur isotope equilibria in the $\mathrm{BaSO}_{4-}$ $\mathrm{HSO}_{4}-\mathrm{H}_{2} \mathrm{O}$ system from 110 to $350{ }^{\circ} \mathrm{C}$ and applications. Geochim. Cosmochim. Acta $41,1033-1040$

Laux, J.H., 1999. Caracterização da mineralização cupro-aurífera de uma parte da mina Uruguai. Rio Grande do Sul. Pós-Graduação em GeologiaUNISINOS, São Leopoldo (Dissertação de Mestrado. 135 pp.)

Laux, J.H., Lindenmayer, Z.G., 2000a. As Minas do Camaquã: um século de evolução de hipóteses genéticas. In: Ronchi, L.H., Lobato, A.O.C. (Coords), Minas do Camaquã, um estudo multidisciplinar. UNISINOS/FAPERGS, (Universidade do Vale do Rio dos Sinos/Fundação de Amparo à Pesquisa do Estado do Rio Grande do Sul) São Leopoldo, pp. 133-164.

Laux, J.H., Lindenmayer, Z.G., 2000b. Caracterização do minério filoneano de um setor da Mina Uruguai, Minas do Camaquã, in: Ronchi, L.H., Lobato, A.O.C. (Coords) Minas do Camaquã, um estudo multidisciplinar. UNISINOS/FAPERGS (Universidade do Vale do Rio dos Sinos/Fundação de Amparo à Pesquisa do Estado do Rio Grande do Sul), São Leopoldo, pp. 191-210.

Laux, J.H., Lindenmayer, Z.G., Teixeira, J.B.G., Neto, A.B., 2005. Ore genesis at the Camaquã copper mine, a Neoproterozoic sediment-hosted deposit in southern Brazil. Ore Geol. Rev. 26, 71-89.

Leites, S.R., Lopes, R.C., Wildner, W., Porcher, C.A., Sander, A., 1990. Divisão litofaciológica da Bacia do Camaquã na Folha Passo do Salsinho, Caçapava do Sul-RS, e sua interpretação paleoambiental. Congr. Bras. Geol. 36 (1), 300-312.

Leiz, V., Almeida, S.C., 1941. Gênese da jazida de cobre "Camaquãm". Município de Caçapava do Sul - Rio Grande do Sul. Secretaria dos negócios de agricultura, indústria e Comércio do Estado do Rio Grande do Sul. Boletim, n ${ }^{\circ} 88$. DNPM (56 pp.).

Li, Y.B., Liu, J.M., 2006. Calculation of sulfur isotope fractionation in sulfides. Geochim. Cosmochim. Acta 70, 1789-1795.

Lima, L. De M., 1998. A Mina Uruguai e Jazida Santa Maria-Distrito Mineiro de Camaquã (RS): Um Estudo Petrológico, Geoquímico e Geotermométrico. Dissertação de Mestrado, programa de Pós-Graduação em GeologiaUniversidade do Vale do Rio dos Sinos, UNISINOS.

Lima, E.F., Nardi, L.V.S., 1992. O magmatismo shoshonítico no estado do Rio Grande do Sul. Uma revisão. Pesquisas 19 (2), 190-194. 
Lima, E.F., Nardi, L.V.S., 1998. O Vulcanismo alcalino e shoshonítico da bacia do Camaquã: Estado do Rio Grande do sul, Brasil. Cong. Urug. de Geol, 2, pp. 263-268.

Lloyd, R.M., 1968. Oxygen isotope behavior in the sulfate-water system. J. Geophys. Res. 73, 6099-6110.

Lüders, V., Pracejus, B., Halbach, P., 2001. Fluid inclusion and sulfur isotope studies in probable modem analogue Kuroko-type ores from the JADE hydrothermal field (Central Okinawa Trough, Japan). Chem. Geol. 173, 45-58.

Nardi, L.V.S., Bitencourt, M. de F., 2009. A-type granitic rocks in post-collisional settings in southernmost Brazil: their classification and relationship with tectonics and magmatic series. Can. Mineral. 47, 1493-1503.

Nardi, L.V.S., Lima, E.F., 1985. A associação shoshonítica de Lavras do Sul, RS. Rev. Bras. Geosci. 15 (2), 139-146.

Nardi, L.V.S., Lima, E.F., 1988. Hidrotermalismo no Complexo Granítico Lavras e Vulcânicas Associadas. Rev. Bras. Geosci. 18 (3), 369-375.

Noguchi, T., Shinjo, R., Ito, M., Takada, J., Oomori, T., 2011. Barite geochemistry from hydrothermal chimneys of the Okinawa Trough: insight into chimney formation and fluid/sediment interaction. J. Mineral. Petrol. Sci. 106, 26-35.

Ohmoto, H., 1986. Stable isotope geochemistry of ore deposits. Rev. Mineral. 16, 491-559.

Ohmoto, H., Goldhaber, M.B., 1997. Sulfur and carbon isotopes, In: Barnes, H.L. (Ed.), Geochemistry of Hydrothermal Ore Deposits, 3rd edition. Wiley, New York, pp. 517-611.

Ohmoto, H., Lasaga, A.C., 1982. Kinetics of reactions between aqueous sulfates and sulfides in hydrothermal systems. Geochim. Cosmochim. Acta 46, 1727-1745.

Ohmoto, H., Rye, R.O., 1979. Isotopes of sulfur and carbon, In: Barnes, H.L. (Ed.), Geochemistry of Hydrothermal Ore Deposits, 2nd edition. Wiley, New York, pp. 509-567.

Oliveira, J.M.M.T., Fernandes, L.A.D., 1991. Estagios finais da deformação do Cinturão Dom Feliciano: Tectonica e sedimentação da Formação Arroio dos Nobres. Simpósio Nacional de Estudos Tectônicos, 3, pp. 1-4.

Paim, P.S.G., Lopes, R.C., 2000. Geologia da região das Minas do Camaquã. In: Ronchi, L.H. and Lobato, A.O.C. (Org). Minas do Camaquã, um estudo multidisciplinar. UNISINOS/ FAPERGS, (Universidade do Vale do Rio dos Sinos/Fundação de Amparo à Pesquisa do Estado do Rio Grande do Sul), São Leopoldo, pp. 111-132.

Paim, P.S.G., Lopes, R.C., Chemale Jr., F., 1995. Aloestratigrafia, sistemas deposicionais e evolução paleogeográfica da Bacia do Camaquã-Vendiano Superior/Ordoviciano Inferior do RS. Simpósio Sul-Brasileiro de Geologia, $6^{\circ}$ Encontro de Geologia do Cone Sul. Resumos, 1. Sociedade Brasileira de Geologia, SBG, Porto Alegre, pp. 39-49.

Paim, P.S.G., Chemale Jr., F., Lopes, R.C., Lopes, R.C., 2000. A Bacia do Camaquã. In: Holz, M., DE ROS, L.F. (Eds.), Geologia do Rio Grande do Sul. Universidade Federal do Rio Grande do Sul, Porto Alegre, pp. 231-274.

Paytan, A., Mearon, S., Cobb, K., Kastner, M., 2002. Origin of marine barite deposits: Sr and S isotope characterization. Geology 30, 747-750.

Philipp, R.P., Massone, H.-J., Theye, T., Campos, R.S., 2009. U-Th-Pb EMPA geochronology of polygenetic monazites of the metapelitic migmatitic gneisses of Camboriú Complex, SC, southern Brazil: evidences or the collisional and post-collisional events in Dom Feliciano Belt. Simpósio 45 anos de Geocronologia no Brasil, São Paulo. Boletim de Resumos Expandidos, pp. 289-291.

Remus, M.V.D., McNaughton, N.J., Hartmann, L.A., Groves, D.I., 1997. Pb and S isotope signature of sulphides and constraints on timing and sources of $\mathrm{Cu}(\mathrm{Au})$ mineralization at the Camaquã and Santa Maria Mines, Caçapava do Sul, southern Brazil. South American Symposium Isotopic Geology (SSAGI), São Paulo, Brasil, Resumo expandido, pp. 253-255.

Remus, M.V.D., McNaughton, N.J., Hartmann, L.A., Koppe, J.C., Fletcher, I.R., Groves, D.I., Pinto, V.M., 1999. Gold in the Neoproterozoic juvenile Bossoroca volcanic arc of southernmost Brazil: isotopic constraints on timing and sources. J. S. Am. Earth Sci. 12, 349-366.

Remus, M.V.D., Hartmann, L.A., McNaughton, N.J., Groves, D.I., Reischl, J.L., 2000. Distal magmatic-hydrothermal origin for the Camaquã $\mathrm{Cu}(\mathrm{Au}-\mathrm{Ag})$ and Santa Maria $\mathrm{Pb}$, $\mathrm{Zn}(\mathrm{Cu}-\mathrm{Ag})$ deposits, southern Brazil. Gondwana Res. 3, 155-174.

Renac, C., Kyser, K., Bowden, P., Moine, B., Cottin, J.Y., 2010. Hydrothermal fluid interaction in basaltic lava units, Kerguelen Archipelago (SW Indian Ocean). Eur. J. Mineral. 22, 215-234.

Ribeiro, M., Fantinel, L.M., 1978. Associações petrotectônicas do Escudo Sul-Riograndense: tabulação e distribuição das associações petrotectônicas do Escudo do Rio Grande do Sul. Iheringia, Série Geológica, 5 19-54

Ribeiro, M.J., Bocchi, P.R., Figueiredo Filho, P.M., Tessari, R., 1966. Geologia da quadrícula de Caçapava do Sul, RS, Brasil. Boletim da Divisão de Fomento da Produção Mineral, 127. DNPM 232.

Richards, J.P., 2009. Postsubduction porphyry $\mathrm{Cu}-\mathrm{Au}$ and epithermal Au deposits: products of remelting of subduction-modified lithosphere. Geology 37, 247-250.

Ronchi, L.H., Lindenmayer, Z.G., Bastos Neto, A., Murta, C.R., 2000. O stockwork e a zona do minério sulfetado no arenito inferior da Mina Uruguai, RS. In: Ronchi L.H., Lobato, A.O.C. (Orgs) Minas do Camaquã, um estudo multidisciplinar. UNISINOS/FAPERGS (Universidade do Vale do Rio dos Sinos/Fundação de Amparo à Pesquisa do Estado do Rio Grande do Sul), São Leopoldo, p. 165-190.

Rowins, S.M., 2000. Reduced porphyry copper-gold deposits: a new variation on an old theme. Geology 28 (6), 491-494.

Rye, R.O., 2005. A review of the stable-isotope geochemistry of sulfate minerals in selected igneous environments and related hydrothermal systems. Chem. Geol. 215, 5-36.
Santos, J.F., Chaban, N., 1978. Concentrações de cobre sedimentar do Tipo red-bed em Caçapava do Sul (RS). Congresso Brasileiro de Geologia, 30. Anais, vol. 6. Sociedade Brasileira de Geologia, SBG, Recife, pp. 2562-2575.

Savin, S.M., Lee, M., 1988. Isotopic studies of phyllosilicates. In: Bailey, S.W. (Ed.), Hydrous phyllosilicates (exclusive of micas) Mineralogical Society of America. Reviews in Mineralogy, 19, pp. 189-219.

Seal II, R.R., 2006. Sulfur isotope geochemistry of sulfide minerals. Rev. Mineral. Geochem 61, 633-677.

Seal II, R.R., Alpers, C.N., Rye, R.O., 2000. Stable isotope systematics in sulfate minerals Rev. Mineral. Geochem. 40, 541-602.

Sheppard, S.M.F., Epstein, S., $1970 . \mathrm{D} / \mathrm{H}$ and ${ }^{18} \mathrm{O} /{ }^{16} \mathrm{O}$ ratios of minerals of possible mantle or lower crustal origin. Earth Planet. Sci. Lett. 9, 232-239.

Simon, A.C., Ripley, E.M., 2011. The role of magmatic sulfur in the formation of ore deposits. Rev. Mineral. Geochem. 73, 513-578.

Sommer, C.A., Lima, E.F., Nardi, LV.S., Liz, J.D., Waichel, B.L., 2006. The evolution of Neoproterozoic magmatism in southernmost Brazil: shoshonitic, high-K tholeiitic and silica-saturated, sodic alkaline volcanism in post-collisional basins. An. Acad Bras. Cienc. 78 (3), 573-589.

Taylor Jr., H.P., 1968. The oxygen isotope geochemistry of igneous rocks. Contr. Mineral. Petrol. 19, 1-71.

Taylor, B.E., 1987. Stable isotope geochemistry of ore-forming fluids. In: Kyser, T.K. (Ed.), Stable isotope geochemistry of low temperature processes. Mineralogical Association of Canada, Short Course Handbook, 13, pp. 337-445.

Teixeira, G., 1978. Conteúdo de Prata e Ouro no minério de cobre nas Minas do Camaquã. Congresso Brasileiro de Geologia, 30. Anais, vol. 4. Sociedade Brasileira de Geologia, SBG, Recife, pp. 1633-1643.

Teixeira, G., Gonzáles, A.P., 1988. Minas do Camaquã, município de Caçapava do Sul, RS Principais Depósitos Minerais do Brasil, vol. III. DNPM 33-41.

Teixeira, G., Gonzales, A.P., Gonzales, M.A., Licht, O.A.B., 1978a. Contribuicão ao estudo de mineralizações cupríferas disseminadas no Distrito Minas do Camaquã. Congresso Brasileiro de Geologia, 30. Anais, vol. 4. Sociedade Brasileira de Geologia, SBG, Recife pp. $1644-1654$.

Teixeira, G., Gonzales, A.P., Gonzales, M.A., Licht, O.A.B., 1978b. Situação das Minas do Camaquã, Rio Grande do Sul. Congresso Brasileiro de Geologia, 30. Anais, vol. 4 Sociedade Brasileira de Geologia, SBG, Recife, pp. 1893-1905.

Torres, M.E., Brumsack, H.J., Bohrmann, G, Emeis, K.C. 1996. Barite fronts in continenta sediments: a new look at barium remobilization in the zone of sulfate reduction and formation of heavy barites in authigenic fronts. Chem. Geol. 127, 125-139.

Torres, M.E., McManus, J., Huh, C.A., 2002. Fluid seepage along the San Clemente Fault scarp: basin-wide impact on barium cycling. Earth Planet. Sci. Lett. 203, 181-194.

Troian, G.C., Mexias, A.S., Gomes, M.E.B., Canarim, D., Patricia Patrier-Mas, P., Renac, C., 2009. Cloritização na Mina Uruguai, Minas do Camaquã, RS, Brasil ISSN 1518-2398 E-ISSN 1807-9806. Pesqui. Geociênc. 37 (3), 173-190.

Veigel, R., 1989. Evolução Diagenética e Mineralização $\mathrm{Cu}-\mathrm{Pb}-\mathrm{Zn}$ dos "Red-Beds" do Distrito de Camaquã - RS. (Dissertação de Mestrado) UnB - Universidade de Brasília (185 pp.).

Veigel, R., 1992. Diagênese de rochas siliciclásticas: O exemplo do Membro Vargas, Eopaleozóico da Bacia do Camaquã - RS. Acta Geol. Leopold. 15, 27-153.

Veigel, R., Dardena, M.A., 1990. Paragênese e sucessão mineral nas diferentes etapas da evolução da mineralização $\mathrm{Cu}-\mathrm{Pb}-\mathrm{Zn}$ do Distrito de Camaquã, RS. Rev. Bras. Geocienc. 20 (1-4), 55-67.

Vidal, O., Parra, T., Trotet, F., 2001. A thermodynamic model for Fe-Mg aluminous chlorite using data from phase equilibrium experiments and natural pelitic assemblages in the 100 to $600{ }^{\circ} \mathrm{C}, 1$ to $25 \mathrm{kbar}$ range. Am. J. Sci. 301 (6), 557-592.

von Damm, K.L., Edmond, J.M., Grant, B., Measures, C.I., Walden, B., Weiss, R.F., 1985 Chemistry of submarine hydrothermal solutions at $21^{\circ} \mathrm{N}$, East Pacific Rise. Geochim. Cosmochim. Acta 49, 2197-2220.

Walker, J.R., 1993. Chlorite polytype geothermometry. Clay Clay Miner. 41, 260-267.

Walker, T.R., Waugh, B., Crone, A.J., 1978. Diagenesis of first-cycle desert alluvium of Cenozoic age, southwestern U.S. and northwestern Mexico. Geol. Soc. Am. Bull. 89, 19-32.

Walshe, J.L., 1986. A six-component chlorite solid solution model and the conditions of chlorite formation in hydrothermal and geothermal systems. Econ. Geol. 81, 681-703.

Wasserman, M.D., Rye, R.O., Bethke, P.M., Arribas Jr., A., 1992. Methods for separation and total stable isotope analysis of alunite. U.S. Geol. Survey. Open-File Rep, pp. 92-99.

Watanabe, M., Sakai, H., 1983. Stable isotope geochemistry of sulfates from the Neogene ore deposits in the Green Tuff region, Japan. Econ. Geol. 5, 282-291.

Wildner, W., Nardi, L.V.S., Lima, E.F., 1999. Post-collisional alkaline magmatism on the Taquarembó Plateau: a well preserved Neoproterozoic-Cambrian plutono-volcanic association in southern Brazil. Int. Geol. Rev. 41, 1082-1098.

Wildner, W., Lima, E.F., Nardi, L.V.S., Sommer, C.A., 2002. Volcanic cycles and setting in the Neoproterozoic III to Ordovician Camaquã Basin succession in southern Brazil: characteristics of post-collisional magmatism. J. Volcanol. Geotherm. Res. 118, 261-283.

Zang, W., Fyfe, W.S., 1995. Chloritization of the hydrothermally altered bedrock at the Igarapé Bahia gold deposits, Carajas, Brazil. Mineral. Deposita 30, 30-38. 